\title{
Mapping ionospheric backscatter measured by the SuperDARN HF radars - Part 1: A new empirical virtual height model
}

\author{
G. Chisham ${ }^{1}$, T. K. Yeoman ${ }^{2}$, and G. J. Sofko ${ }^{3}$ \\ ${ }^{1}$ British Antarctic Survey, Natural Environment Research Council, High Cross, Madingley Road, Cambridge, CB3 0ET, UK \\ ${ }^{2}$ Department of Physics and Astronomy, University of Leicester, Leicester, LE1 7RH, UK \\ ${ }^{3}$ University of Saskatchewan, Saskatoon, S7N 5E2, Canada
}

Received: 17 August 2007 - Revised: 29 January 2008 - Accepted: 6 March 2008 - Published: 13 May 2008

\begin{abstract}
Accurately mapping the location of ionospheric backscatter targets (density irregularities) identified by the Super Dual Auroral Radar Network (SuperDARN) HF radars can be a major problem, particularly at far ranges for which the radio propagation paths are longer and more uncertain. Assessing and increasing the accuracy of the mapping of scattering locations is crucial for the measurement of two-dimensional velocity structures on the small and mesoscale, for which overlapping velocity measurements from two radars need to be combined, and for studies in which SuperDARN data are used in conjunction with measurements from other instruments. The co-ordinates of scattering locations are presently estimated using a combination of the measured range and a model virtual height, assuming a straight line virtual propagation path. By studying elevation angle of arrival information of backscatterred signals from 5 years of data (1997-2001) from the Saskatoon SuperDARN radar we have determined the actual distribution of the backscatter target locations in range-virtual height space. This has allowed the derivation of a new empirical virtual height model that allows for a more accurate mapping of the locations of backscatter targets.
\end{abstract}

Keywords. Ionosphere (Active experiments; Wave propagation; Instruments and techniques)

\section{Introduction}

Coherent scatter radars are one of the most successful instruments used for probing dynamical processes in the Earth's ionosphere. They measure the motion of magnetic fieldaligned ionospheric density irregularities in the ionospheric E- and F-regions which act as backscatter targets for the transmitted radio signals. One necessary condition for the

Correspondence to: G. Chisham

(gchi@bas.ac.uk) production of coherent backscatter is that the wave vector of the radio signal is propagating orthogonal to the Earth's magnetic field at the point of scatter (Bates and Albee, 1970). For ultra high frequency (UHF) and very high frequency (VHF) radio signals, refraction of the signal is generally weak or nonexistent and signal propagation in the Earth's ionosphere is generally along straight line paths. At low and middle latitudes these rectilinear rays are highly suitable for studying the ionosphere (especially the E-region ionosphere where it is easier for these rays to achieve orthogonality with the magnetic field). However, at high latitudes where the magnetic field becomes increasingly vertical, it becomes progressively more difficult for rectilinear rays to achieve orthogonality with the Earth's magnetic field, especially at higher altitudes such as in the F-region ionosphere. In contrast to UHF and VHF signals, high frequency (HF) radio signals are very susceptible to refractive effects (Weaver, 1965), and hence, variations in ionospheric electron density play a major role in $\mathrm{HF}$ propagation. Consequently, measurements of irregularities in the high-latitude ionospheric F-region are best made by radars which transmit in the $\mathrm{HF}$ range $(3-30 \mathrm{MHz})$, where the refraction of signals by the ionosphere allows them to achieve orthogonality with the Earth's magnetic field over a wider range of altitudes, and hence, to backscatter off fieldaligned density irregularities in both the E- and F-region ionosphere.

The Super Dual Auroral Radar Network (SuperDARN) (Greenwald et al., 1995) is a network of ground-based coherent-scatter radars that operate in the HF band and whose fields of view combine to cover extensive regions of both the Northern and Southern Hemisphere polar ionospheres. Over the last decade SuperDARN has been one of the most successful tools for studying high-latitude plasma convection and large-scale dynamical processes in the Earth's magnetosphere, ionosphere, and upper atmosphere (Chisham et al., 2007). This wide range of scientific studies is made possible because the F-region density

Published by Copernicus Publications on behalf of the European Geosciences Union. 
irregularities that act as backscatter targets drift with the background plasma motion (Villain et al., 1985; Ruohoniemi et al., 1987). Hence, when irregularities are present, SuperDARN can image ionospheric convection across the polar ionospheres.

Each SuperDARN radar is an electronically-steerable, narrow-beam, phased-array radar that comprises a main array of $16 \log$-periodic antennae. The standard 16-beam scan employed by the radars creates individual fields of view that typically extend $\sim 52^{\circ}$ in azimuth, and from $\sim 200 \mathrm{~km}$ to more than $3000 \mathrm{~km}$ in range. The SuperDARN radars are frequency agile and can operate over a wide range of $\mathrm{HF}$ frequencies (from 8 to $20 \mathrm{MHz}$ ). This frequency can be adjusted to find the best propagation paths to irregularity regions where the signals can be backscattered. The radars transmit a multi-pulse sequence and the backscattered signals (or echoes) they receive are sampled and processed to produce multi-lag complex autocorrelation functions (ACFs) at all ranges. The ACFs are fitted by standard functions to estimate the backscattered power, the line-of-sight Doppler velocity of the irregularities, and the width of the Doppler power spectrum for each range where there are significant returns (Hanuise et al., 1993; Baker et al., 1995). In addition to the main antenna array, many SuperDARN radars have an interferometer array of 4 antennae, located some distance either in front of, or behind, the main array. Determination of the cross-correlation function (XCF) of the signals received at the main and interferometer arrays allows the measurement of the phase difference between the backscattered signals measured by the two arrays. Knowledge of this phase difference allows the determination of the elevation angle of arrival of the backscattered signals (Milan et al., 1997; André et al., 1998). However, the range of measurable elevation angles is limited by the $2 \pi$ ambiguity of the phase measurements and depends on the operational frequency and the distance between the two antenna arrays.

One of the most important aspects of SuperDARN data analysis is mapping the locations of the scattering regions, specifically determining their geographic/geomagnetic coordinates. The required accuracy of this mapping depends on the application of the measurements. The resolution of features in convection maps determined using large-scale $\mathrm{Su}$ perDARN global convection mapping is typically limited to hundreds of $\mathrm{km}$ and hence, small uncertainties in mapping have little influence on these convection maps. However, assessing and increasing the accuracy of this mapping is crucial for the following types of investigation:

- Studies of small and meso-scale velocity structures, where overlapping velocity measurements from two $\mathrm{Su}-$ perDARN radars need to be combined to determine two-dimensional velocity vectors (e.g. Huang et al., 2000; Chisham et al., 2000).

- Comparisons between measurements from SuperDARN and space-based instruments, e.g. auroral images,
DMSP particle precipitation measurements, etc. (e.g. Chisham et al., 2005; Hubert et al., 2006).

- Comparisons between measurements from SuperDARN and other ground-based instruments, e.g. EISCAT, SPEAR, etc. (e.g. Woodfield et al., 2002; Senior et al., 2004).

Accurate mapping of scattering regions requires knowledge of the HF signal propagation paths. The propagation paths of signals backscattered from ionospheric irregularities (termed ionospheric backscatter) can be classified in the most simple terms as $\frac{1}{2}$-hop (direct to ionosphere and back again) or $1 \frac{1}{2}$ hop (to the ionosphere, to the ground, and to the ionosphere and back again). Longer paths (e.g. $2 \frac{1}{2}$-hop) are possible but make up a very small percentage of the SuperDARN data set.

The exact propagation paths of HF rays depend heavily on both horizontal and vertical ionospheric electron density variations which can be very complex and variable, especially in the high-latitude F-region (e.g. Vickrey et al., 1980). An important technique for understanding radio propagation is ray tracing (e.g. Jones and Stephenson, 1975) which involves tracing the paths of radio waves through the ionosphere assuming a particular ionospheric electron density model. This technique has been used extensively but typically to study the basic features of propagation through a simplified ionosphere. Villain et al. (1984) used ray tracing to study HF propagation paths in a more realistic model of the high-latitude ionosphere. They showed that the sensitivity of propagation to density changes means that accurately determining propagation paths is difficult, even with more sophisticated models. Their results showed significant differences in F-region propagation paths between simple and more realistic ionospheric models and are a good illustration of the problems of accurately mapping the scattering region. Hence, a wholly accurate mapping of the location of the scattering volume requires detailed spatiotemporal measurements of the ionospheric electron density (which are rare). Currently, in the absence of accurate and reliable measurements or models of ionospheric electron density variations, a simple algorithm is used to map the scattering locations of SuperDARN backscatter.

The simplest scenario that can be considered when mapping HF propagation paths is that of a flat Earth. Figure 1a presents a simplified diagram which shows a $\frac{1}{2}$-hop backscatter ray path for the assumption of a flat Earth and a planar ionosphere. The blue square highlights the location of the radar and the dashed black arrow represents a line perpendicular to the Earth's surface which contains the scattering location. The ground distance between these two points is the ground range. The black line represents a virtual ray path from the radar site to a virtual scattering point (i.e. the straight line propagation path that would result if there was no refraction of the ray by the ionosphere). The distance along this path is the range. The angle between the virtual 
(a)

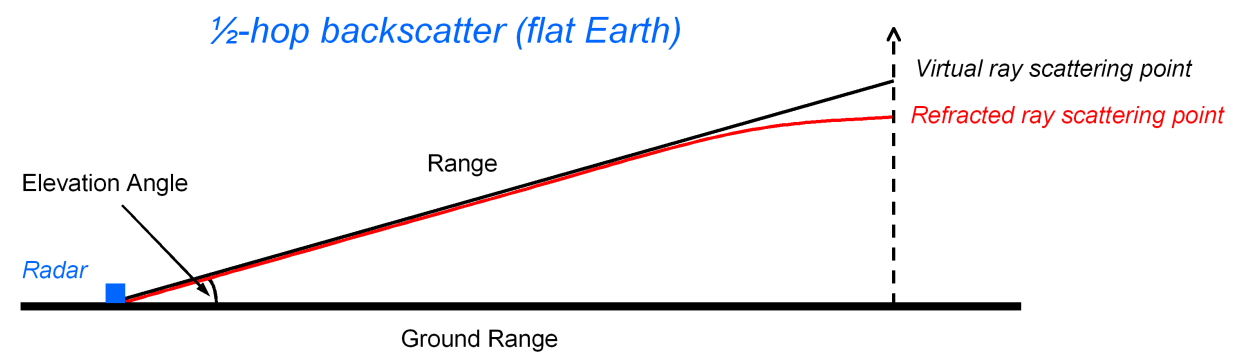

(b)

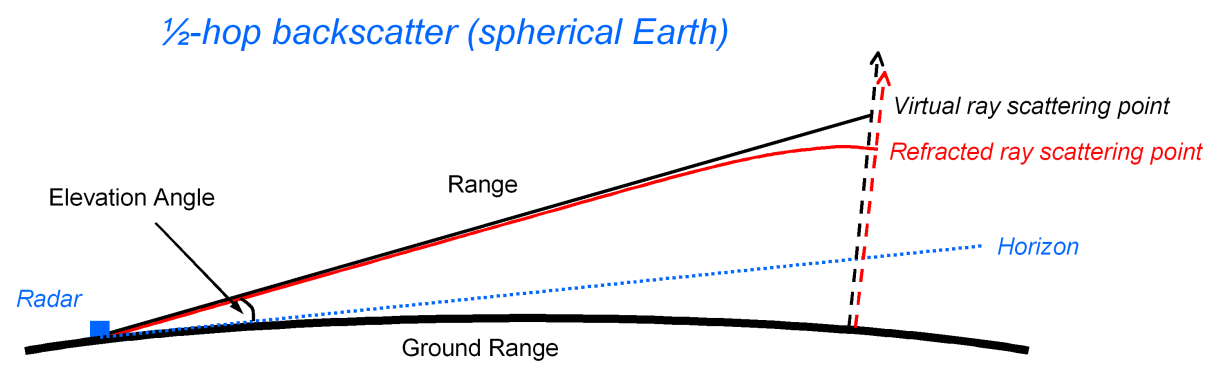

(c)

\section{1/2-hop backscatter (spherical Earth)}

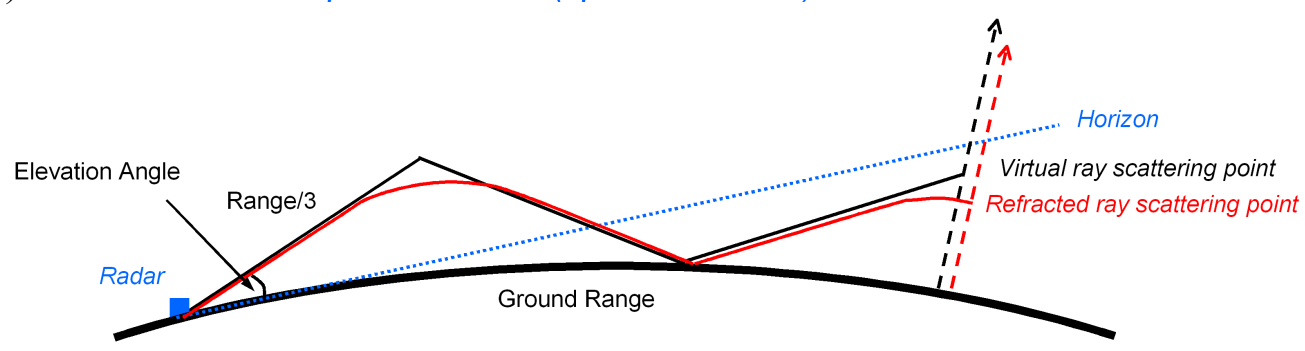

Fig. 1. Simple schematic diagrams illustrating refracted and virtual HF propagation paths for (a) $\frac{1}{2}$-hop ionospheric backscatter assuming a flat Earth, (b) $\frac{1}{2}$-hop ionospheric backscatter assuming a spherical Earth, and (c) $1 \frac{1}{2}$-hop ionospheric backscatter assuming a spherical Earth. In all panels the bold black line represents the surface of the Earth in the plane of the propagation path, the solid black line represents the virtual propagation path, the solid red line represents the refracted propagation path, the dashed black and red lines are perpendicular to the Earth's surface at the virtual and refracted ray scattering points, respectively, and the dotted blue line represents the horizon at the radar site. The elevation angle is the angle between the virtual path and the horizon.

path and the horizon is the elevation angle. The red line represents an estimate of a real ray path which is refracted by the increasing electron density in the ionosphere, resulting in a true scattering point, where the ray is orthogonal to the local magnetic field, which is at a lower altitude than the associated virtual scattering point. This representation assumes that the ray travels along the same path both to and from the scattering location, which assumes that the ionosphere is invariant during the travel time. Breit and Tuve (1926) first showed that when a flat Earth and planar ionosphere are assumed the real and virtual propagation paths have the same ground range (i.e. the virtual scattering point for any ray is located directly above its real scattering point).
The mapping of scattering locations is trivial for the case of a flat Earth and planar ionosphere if both the range and elevation angle of the ray are known. All the backscattered signals measured by the SuperDARN radars have an associated range and many have measurements of the elevation angle as well. This allows an exact determination of the ground range from the radar to the ground location of the perpendicular which passes through both the virtual and refracted ray scattering points. However, in many cases reliable elevation angle measurements are not available. In addition, some of the SuperDARN radars do not have the interferometer arrays which provide the capability to measure the elevation angle. Estimating the virtual scattering location in the absence of an elevation angle measurement requires the use of a virtual 
height model that provides an estimate of the virtual height of the scattering location for any given range. Hence, the accuracy of the ground range determination (and hence the mapping) in this case is wholly dependent on the accuracy of the assumed virtual height.

For most propagation paths longer than a few hundreds of $\mathrm{km}$ the flat Earth approximation is poor and a spherical Earth must be assumed. Figure $1 \mathrm{~b}$ shows a simplified diagram explaining the $\frac{1}{2}$-hop backscatter ray path when assuming a spherical Earth. For a spherical Earth, differences between virtual and refracted propagation paths mean that the ground ranges to the virtual and real scattering points are slightly different (i.e. the Breit-Tuve theorem for propagation above a flat Earth does not hold for a spherical Earth). In Fig. $1 b$ the dashed black arrow shows the perpendicular passing through the virtual scattering point and the red arrow shows the perpendicular passing through the real scattering point. The dotted blue line represents the horizon at the radar site. The mapping of scattering locations is more difficult for the case of a spherical Earth. Knowledge of both the range and elevation angle allows the calculation of the ground range to the virtual scattering location. As in the case of a flat Earth a lack of elevation angle measurements requires the use of a virtual height model to estimate this ground range. However, as shown schematically in Fig. 1, for the case of a spherical Earth a ground range offset exists between the real and virtual scattering locations. However, by making the assumption that the electron density within the ionosphere is spherically uniform and varies only with altitude, this difference can be assumed to be small (especially at near ranges). Ray-tracing studies suggest that for typical ionospheric conditions the actual ground range to the scattering region may be $\sim 15-60 \mathrm{~km}$ different from that predicted from using the virtual path (e.g. Baker et al., 1986; André et al., 1997). This limitation on the accuracy of the measurement of the ground range of the scattering location is presently difficult to overcome.

Figure 1c shows the scenario for $1 \frac{1}{2}$-hop backscatter. Here, rays are refracted by the ionosphere and travel back towards the ground (1-hop) from where they are reflected back up towards the ionosphere ( $1 \frac{1}{2}$-hop). Here, they are scattered back along the same path. Assuming a spherically uniform ionosphere, and that the maximum altitude of the first hop path is the same as the altitude of the final scattering point, the virtual path for any refracted ray path can be approximated as three connected line segments of the same length all having the same elevation angle at the point at which they connect to the ground. Each line segment has a length which is a third of the total range. In this case, the locations of both the real and virtual scattering points can be "over the horizon". Because of the longer ray paths the difference between the ground range to the virtual and real scattering points is likely to be larger than in the $\frac{1}{2}$-hop backscatter scenario.

At present the standard method for mapping the scattering locations of SuperDARN backscatter makes use of a virtual height model, and elevation angle information is rarely used. The standard method also makes the assumption that the real and virtual propagation paths have approximately the same ground range (i.e. that the perpendicular that passes through the virtual scattering point for any ray is located close to that which passes through the real scattering point, as is the case for the flat Earth assumption). Hence, the largest errors in mapping are thought to result from the inadequacies of the standard virtual height model that is assumed. The following virtual height model is presently the standard model used for SuperDARN backscatter:

$h_{v}=\left\{\begin{array}{lr}\frac{115 r}{150} & \text { for } 0<r<150 \mathrm{~km} \\ 115 & \text { for } 150 \leq r \leq 600 \mathrm{~km} \\ \frac{r-600}{200}\left(h_{i}-115\right)+115 \text { for } 600<r<800 \mathrm{~km} \\ h_{i} & \text { for } r \geq 800 \mathrm{~km}\end{array}\right.$

where $r$ is the range (in $\mathrm{km}$ ), $h_{v}$ is the virtual height (in $\mathrm{km}$ ), and $h_{i}$ is a user-defined maximum virtual height (in $\mathrm{km}$ ), which is typically taken as $400 \mathrm{~km}$. A $\frac{1}{2}$-hop virtual propagation path is then determined using $h_{v}$ and $r$ and assuming straight line propagation to the virtual scattering point. The latitude and longitude of the scattering location are then determined by using the assumption discussed above that the virtual scattering point is vertically above the real scattering point. As a single virtual height value $\left(h_{i}\right)$ is assumed for most F-region backscatter, and because a $\frac{1}{2}$-hop virtual path is always assumed, there is often uncertainty in the accuracy of the estimated virtual paths and hence in the mapping of the scattering locations, especially at far ranges where $1 \frac{1}{2}$ hop backscatter typically occurs. An additional complication is that the virtual height enters into the determination of the beam direction (because of the cone angle effect) (Milan et al., 1997; André et al., 1998), and hence having an accurate virtual height model is of increased importance.

A number of studies have attempted to assess the accuracy of the SuperDARN algorithm and the associated SuperDARN virtual height model for mapping scattering locations. These studies have sometimes used ray-tracing simulations to investigate possible HF propagation paths (Villain et al., 1984; Baker et al., 1986), or have used velocity cross-correlation between signals from the same radar at different frequencies (André et al., 1997). Yeoman et al. (2001) made use of artificially-induced ionospheric irregularities, as well as the ray-tracing technique, to test the $\mathrm{Su}$ perDARN algorithm. The irregularities were induced using the EISCAT ionospheric heating facility located at Troms $\varnothing$ (Rietveld et al., 1993) and hence provided a definite ground range calibration. The results of Yeoman et al. (2001) suggested that typical ground range errors were of $\sim 16 \mathrm{~km}$ for $\frac{1}{2}$-hop F-region backscatter and $\sim 60 \mathrm{~km}$ for $1 \frac{1}{2}$-hop F-region backscatter. The companion paper to our present study (Yeoman et al., 2008) presents more recent results which show that the typical ground range errors are larger than this and that the standard SuperDARN virtual height model is 
inadequate for accurately mapping scattering locations at far ranges.

In this study, we use 5 years (1997-2001) of elevation angle data from the Saskatoon SuperDARN radar to determine the statistical distribution of scattering locations in rangevirtual height space. We use this statistical virtual height distribution to determine an empirical virtual height model, the accuracy of which can be compared with the standard SuperDARN virtual height model. In the companion paper (Yeoman et al., 2008) we compare the accuracy of these different virtual height models using artificially-induced ionospheric backscatter from both the Troms $\varnothing$ and SPEAR ionospheric heating facilities.

\section{Results}

For this study we have chosen to use elevation angle data from the Saskatoon SuperDARN radar. A large proportion of the backscatter measured by the Saskatoon radar have reliable elevation angle measurements with a good coverage in range, frequency, and magnetic local time (MLT), and with only a small percentage of ground (non-ionospheric) backscatter. We initially use data from Saskatoon beam 3 only, which is aligned close to the geomagnetic meridional direction, but later we compare these results with data from three other Saskatoon beams. We use only common mode data (characterised by $180 \mathrm{~km}$ to the first range gate and a $45 \mathrm{~km}$ range gate separation) in order to simplify the data analysis and the method of presentation of the results. We use 5 years of data (1997-2001 inclusive) which are the five years with the largest rates of backscatter (being centred close to solar maximum in the sunspot cycle).

\subsection{Backscatter statistics}

Figure 2 presents statistical histograms of certain variables in the Saskatoon beam 3 data set, namely range, elevation angle, frequency, and MLT. Here, we separate all the backscatter with a line-of-sight velocity magnitude greater than $100 \mathrm{~m} / \mathrm{s}$ (black histograms, which we assume to be exclusively ionospheric backscatter), from all the backscatter with a line-of-sight velocity magnitude less than $100 \mathrm{~m} / \mathrm{s}$ (red histograms, which we assume to be a mixture of ground and ionospheric backscatter). The presence of significant amounts of ground backscatter in the data set would contaminate our elevation angle analysis. Ground backscatter is characterised by low Doppler velocity $(<\sim 50 \mathrm{~m} / \mathrm{s})$ and low Doppler spectral width $(<\sim 50 \mathrm{~m} / \mathrm{s})$ but is difficult to identify unambiguously. Hence, to ensure a minimal amount of ground backscatter in the ensuing analysis we remove all backscatter with a line-of-sight velocity below the (conservative) velocity threshold of $100 \mathrm{~m} / \mathrm{s}$ (the red histogram). This also removes a large amount of real ionospheric backscatter from the statistical analyses, but it guarantees that the re-
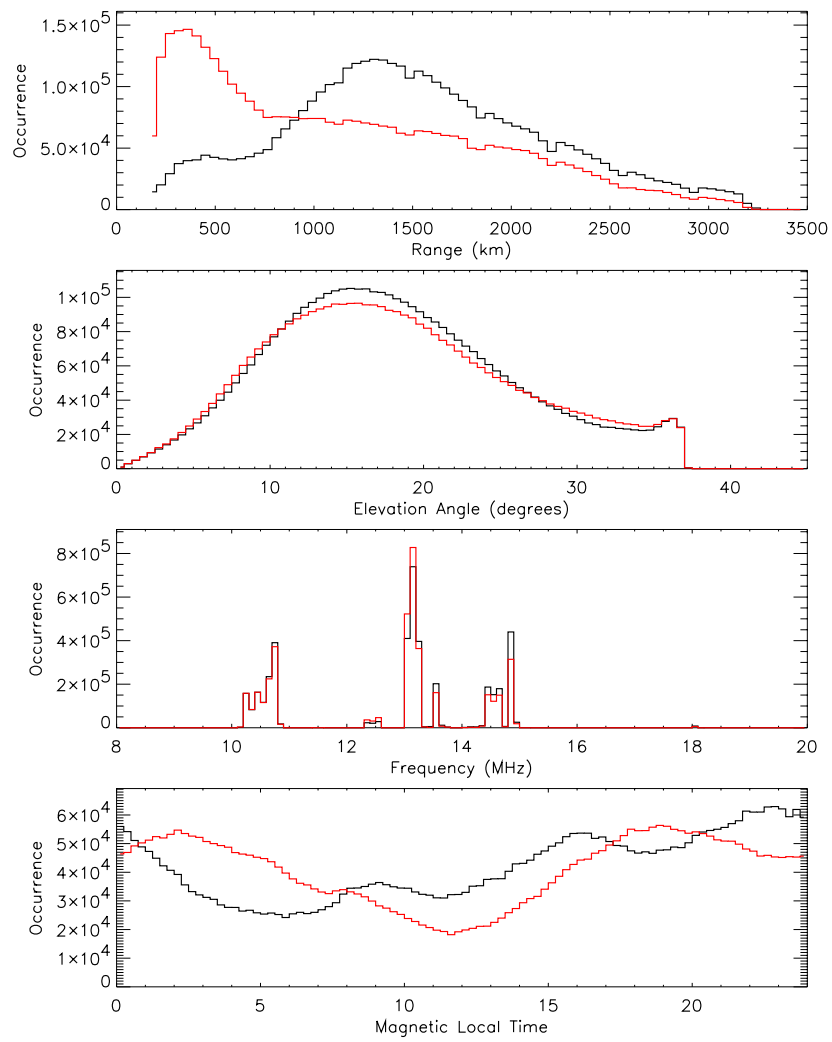

Fig. 2. Statistical histograms of range, elevation angle, frequency, and magnetic local time for Saskatoon beam 3 common mode data from 1997-2001 inclusive. The black curves show the histograms when only backscatter with a line-of-sight velocity magnitude greater than $100 \mathrm{~m} / \mathrm{s}$ are considered. The red curves show the histograms when only backscatter with a line-of-sight velocity magnitude less than $100 \mathrm{~m} / \mathrm{s}$ are considered.

maining backscatter (the black histograms) is not contaminated with ground backscatter, and is justified as long as the number of remaining data samples is adequate for the statistical analysis.

The top panel of Fig. 2 presents the histograms of range, the sampling interval being every range gate $(45 \mathrm{~km}$ range sampling). Looking first at the black curve, there are more than 20000 samples at nearly all ranges with a maximum of $\sim 120000$ samples at $\sim 1300 \mathrm{~km}$ range in the $\frac{1}{2}$-hop Fregion backscatter region. This means that there is enough data for a reliable statistical analysis at every range gate up to $\sim 3200 \mathrm{~km}$ range. The red curve has a large peak at small ranges $(\sim 350 \mathrm{~km})$ which corresponds to low velocity E-region backscatter. This highlights the large amount of low-velocity ionospheric backscatter that is also being removed along with the contaminating ground backscatter.

The second panel of Fig. 2 presents the elevation angle histograms. The distribution of elevation angles is determined partly by the vertical radiation pattern formed by the SuperDARN antennae which will peak at a particular elevation 


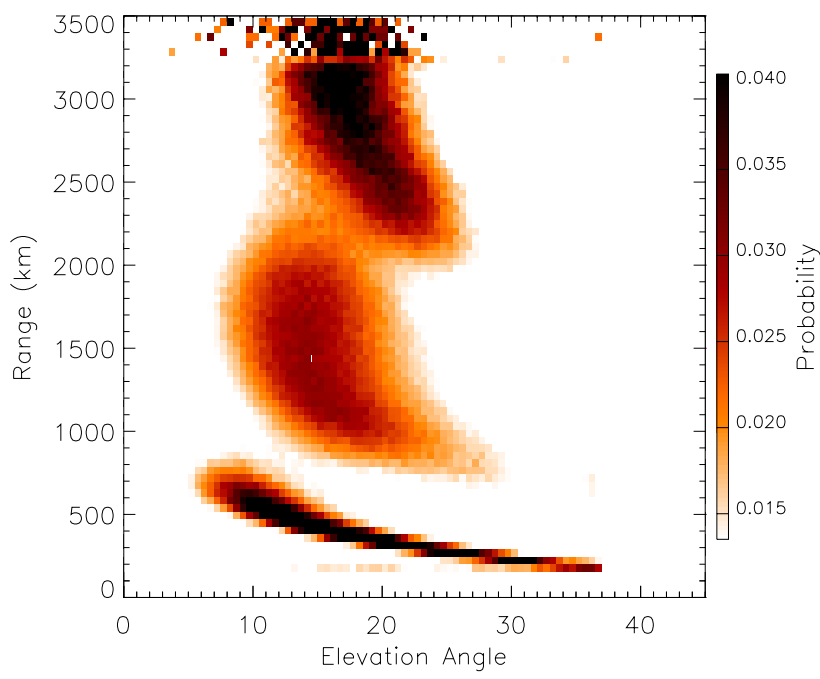

Fig. 3. The distribution of Saskatoon beam 3 backscatter from 1997-2001 inclusive in range-elevation angle space. Only common mode backscatter with a line-of-sight velocity magnitude greater than $100 \mathrm{~m} / \mathrm{s}$ are included. The distribution in each range bin $(45 \mathrm{~km})$ has been normalised. The colour scale provides a key to the occurrence probability in each region; occurrence probabilities of less than 0.014 are shown as white space.

angle for a particular operational frequency and beam orientation; the elevation angle of arrival being the same as the takeoff angle assuming identical propagation paths to and from the scattering region. Here, the two histograms (red and black) are almost identical, both peaking around $15^{\circ}$ elevation.

The third panel of Fig. 2 presents the operational frequency histograms. The operational frequencies used by the SuperDARN radars are varied with the time of day, and with the geomagnetic conditions, in order to optimise the HF propagation path to give the best opportunity of achieving orthogonality of the HF signal with any existing field-aligned irregularities. (However, the frequency bands available for operation for each SuperDARN radar are limited and this is reflected in the histogram.) Lower frequency signals refract in lower electron density regions than higher frequency signals and hence refract more at lower ionospheric altitudes. As higher frequency signals refract less they may not refract enough to achieve orthogonality with the magnetic field at all and hence may continue to penetrate the ionosphere without backscattering. Due to the variable propagation paths that exist for different frequency signals, the virtual height variations with range may vary slightly with operational frequency. This will be considered later in our analysis by splitting the results into different frequency bands. Here, the two histograms (red and black) show very similar distributions with frequency, with significant peaks around 10.6 MHz, 13.2 MHz, and 14.5 MHz, representing the limited bands of operation of the Saskatoon radar.
The final panel of Fig. 2 presents the MLT histograms (at $0.25 \mathrm{~h}$ resolution). Here, the black histogram is greater than 25000 samples at all MLTs illustrating that there is good sampling at all MLTs and unlikely to be any significant MLT bias in the results (at least when considering all frequencies together). The differences between the black and the red histograms are most probably due to the black histogram being dominated by F-region backscatter and the red histogram being dominated by E-region backscatter.

\subsection{Backscatter distribution in range-elevation angle space}

Figure 3 presents the statistical distribution of the Saskatoon beam 3 data set in range-elevation angle space, at a resolution of $45 \mathrm{~km}$ ( 1 common mode range gate) in range and $0.5^{\circ}$ in elevation angle. The darker (lighter) shaded regions represent regions of high (low) probability. The elevation angle distribution in each range gate bin has been normalised in order to be able to clearly visualise the distribution at ranges where the backscatter occurrence is low. As shown by the colour scale, the distribution below an occurrence probability of 0.014 is not shown (white shaded regions) so as to clearly highlight the major regions of the occurrence distribution.

Three distinct distributions of backscatter are evident in Fig. 3. Since ground backscatter has effectively been removed from the data set, these three distributions are most likely to represent $\frac{1}{2}$-hop E-region backscatter (at lowest ranges), $\frac{1}{2}$-hop F-region backscatter (at mid ranges), and $1 \frac{1}{2}$-hop F-region backscatter (at far ranges). However, the transitions between these different distributions do not occur cleanly at specific ranges. In particular, there are a number of range gates over which the $\frac{1}{2}$-hop F-region and $1 \frac{1}{2}$-hop F-region backscatter distributions both have a significant occurrence probability ( $1900-2400 \mathrm{~km}$ range). This figure shows that in these regions it is impossible to determine the propagation mode to, or virtual height of, any scattering region based solely on the range measurement. However, in practice it is often possible to accurately determine the propagation mode based on other information. Firstly, the addition of the elevation angle information makes determination of the propagation mode possible. Secondly, inspection of the measured backscatter across the complete radar field-ofview can allow the categorisation of regions of backscatter into $\frac{1}{2}$-hop, ground, and $1 \frac{1}{2}$-hop.

\subsection{Range-virtual height distribution in real space}

By making assumptions about the mode of propagation $\left(\frac{1}{2}\right.$ hop or $1 \frac{1}{2}$-hop) it is possible to convert the distribution presented in Fig. 3 into a real-space virtual height distribution. If we assume initially that all backscatter results from a $\frac{1}{2}$ hop propagation path (which only applies in reality to the two lower-range distributions in Fig. 3), and we also assume a spherical Earth, it is possible to determine the virtual height $h_{1}$ of each pixel in Fig. 3 with elevation angle $\alpha$ and range 
$r$. We assume a triangle with sides $R_{E}$ (distance from the centre of the Earth to the radar site), $R_{E}+h_{1}(r, \alpha)$ (distance from the centre of the Earth to the virtual scattering point), and $r$ (range of virtual propagation path), and an angle opposite side $R_{E}+h_{1}(r, \alpha)$ of $\alpha+\pi / 2$. Hence, the virtual height can be expressed as

$h_{1}(r, \alpha)=\left(R_{E}^{2}+r^{2}+2 r R_{E} \sin \alpha\right)^{\frac{1}{2}}-R_{E}$

In Fig. 4a we present the distribution of virtual height determined using Eq. (2). The figure shows the curved surface of a spherical Earth with the ground range from the radar marked every $100 \mathrm{~km}$ along this surface. The radar site is located on this surface at $0 \mathrm{~km}$ ground range. The curved blue dashed lines represent lines of constant altitude above the Earth's surface, separated every $200 \mathrm{~km}$. The black dotted lines represent radial lines which pass through the centre of the Earth and are spaced so that they cross the Earth's surface at intervals of $500 \mathrm{~km}$ ground range. The colour scale for the distribution contours is the same as in Fig. 3. As in Fig. 3, the three distinct distributions of backscatter are very clear. However, only the two distributions at lower ranges correspond to $\frac{1}{2}$-hop backscatter and so we only discuss these two distributions here. The lowest range distribution, corresponding to $\frac{1}{2}$-hop E-region backscatter extends over ground ranges of $\sim 100-800 \mathrm{~km}$ with the peak of the distribution occurring at virtual heights $\sim 120-140 \mathrm{~km}$. The mid-range distribution, corresponding to $\frac{1}{2}$-hop F-region backscatter extends over ground ranges of $\sim 600-2200 \mathrm{~km}$ with the peak of the distribution occurring at virtual heights of $\sim 400 \mathrm{~km}$ at lower ranges, increasing to virtual heights of $\sim 800 \mathrm{~km}$ at farther ranges.

This large increase in the $\frac{1}{2}$-hop F-region peak virtual height with range provides important information about the real propagation paths at these ranges. If the real scattering points for our HF signals are near the F-region electron density peak at $\sim 250-400 \mathrm{~km}$ then this suggests that the major mode of propagation for those signals with virtual heights of $\sim 800 \mathrm{~km}$ is in the form of the "Pedersen ray". Pedersen rays represent rays that are at the limit between reflection and penetration. They are HF rays that refract to close to the horizontal in the F-region and are effectively trapped in an ionospheric waveguide. They can therefore travel larger distances within the ionosphere, being capable of reaching F-region scatterers which are far from the radar. Ray-tracing simulations (Baker et al., 1986; Huang et al., 1998b) have shown Pedersen rays that are perpendicular to the magnetic field in the F-region over a large range interval extending from $\sim 900 \mathrm{~km}$ to $\sim 1800 \mathrm{~km}$. Baker et al. (1986) suggested that these rays existed for elevation angles $\sim 12-15^{\circ}$ or less (for a signal frequency of $14.5 \mathrm{MHz}$ ). Additionally, the raytracing analysis of Villain et al. (1984) predicted Pedersenray-like signals that achieved orthogonality with the magnetic field in the F-region over a range of altitudes from $\sim 300-450 \mathrm{~km}$ with $\frac{1}{2}$-hop propagation paths extending to

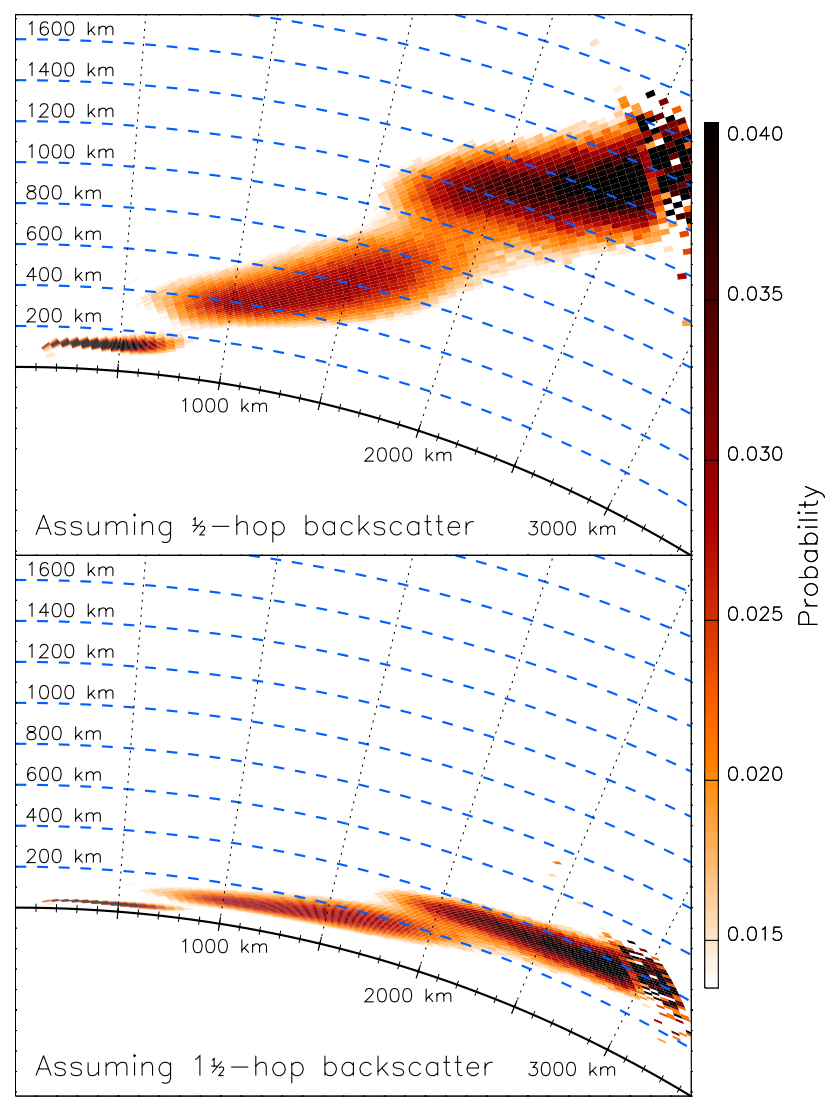

Fig. 4. The distribution of Saskatoon beam 3 backscatter from 1997-2001 inclusive transformed from range-elevation angle space to virtual height in real space by assuming a straight line virtual propagation path. The top panel presents the distribution when a $\frac{1}{2}$-hop propagation path is assumed, as in Fig. $1 \mathrm{~b}$. The bottom panel presents the distribution when a $1 \frac{1}{2}$-hop propagation path is assumed, as in Fig. 1c. Only common mode backscatter with a lineof-sight velocity magnitude greater than $100 \mathrm{~m} / \mathrm{s}$ are included. The distribution in each range bin $(45 \mathrm{~km})$ has been normalised as in Fig. 3. The colour scale provides a key to the occurrence probability in each region; occurrence probabilities of less than 0.014 are shown as white space.

$\sim 2000 \mathrm{~km}$ ground range (for a $16^{\circ}$ elevation angle ray path). These results are consistent with the statistics presented in Figs. 3 and 4.

To study the far range distribution in the same way as the other two distributions we now assume that all backscatter results from a $1 \frac{1}{2}$-hop propagation path (which only applies in reality to the far range distribution in Fig. 3). Again we assume a spherical Earth, and determine the virtual height $h_{2}$ of each pixel in Fig. 3 with elevation angle $\alpha$ and range $r$. Considering an ideal virtual propagation path for $1 \frac{1}{2}$-hop backscatter, the HF ray makes 3 journeys between the Earth and ionosphere and first reaches virtual height $h_{2}$ after a third of the range distance has been covered. This is making the assumption that the maximum virtual height on the initial Earth 


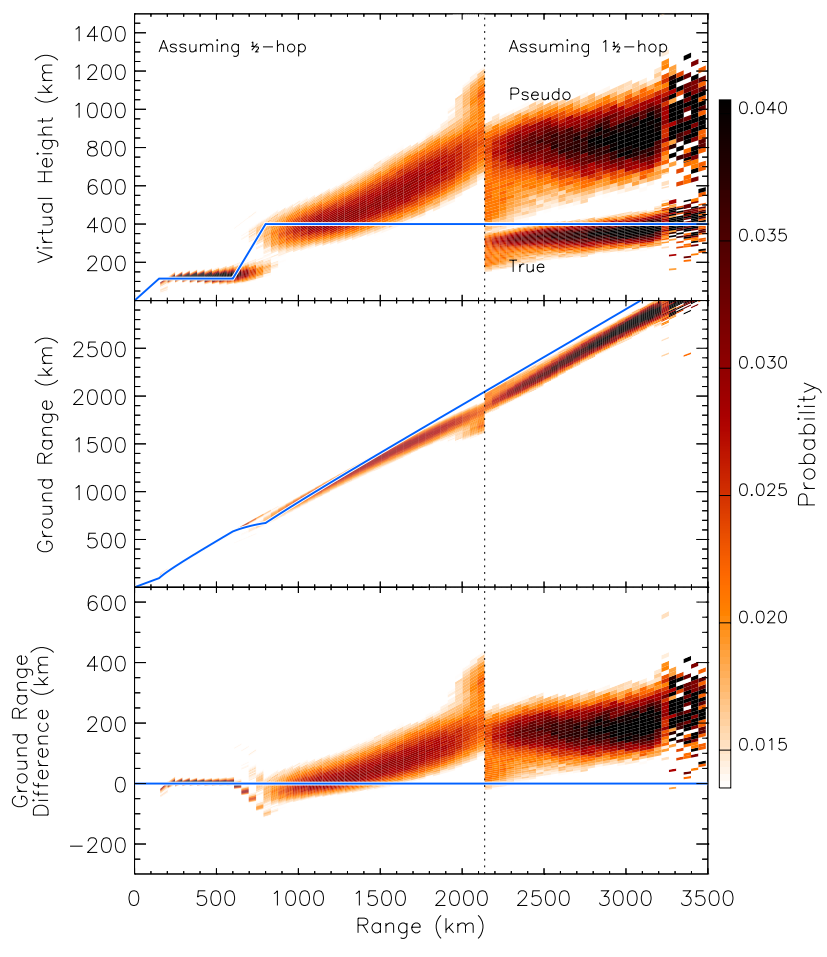

Fig. 5. A comparison of the variation of virtual height and ground range with range between the measured Saskatoon beam 3 backscatter distribution (colour contours) and the standard SuperDARN virtual height model (blue line). (top) The virtual height distribution of Saskatoon beam 3 backscatter from 1997-2001 inclusive (adapted from Fig. 4). $\frac{1}{2}$-hop backscatter is assumed up to a range of $2135 \mathrm{~km}$ (vertical dotted line), whereas $1 \frac{1}{2}$-hop backscatter is assumed at higher ranges. At higher ranges two distributions are shown. The "True" virtual height distribution is the same as in the lower panel of Fig. 4. The "Pseudo" virtual height distribution is the distribution of virtual heights that need to be assumed for $1 \frac{1}{2}$-hop backscatter in order to retain the correct ground range when using a $\frac{1}{2}$-hop backscatter assumption for estimating the ground range. (middle) The ground range distribution for the same data set. (bottom) The ground range difference distribution showing the range of errors that result from application of the SuperDARN standard virtual height model. Here, the blue line represents the measured distribution and the contours represent the differences that occur from using the standard virtual height model. (all panels) Only common mode backscatter with a line-of-sight velocity magnitude greater than $100 \mathrm{~m} / \mathrm{s}$ are included. The distribution in each range bin $(45 \mathrm{~km})$ has been normalised. The colour scale provides a key to the occurrence probability in each region; occurrence probabilities of less than 0.014 are shown as white space.

to ionosphere path is the same as that of the final scattering point. Hence, considering the first third of the virtual propagation path only, we can assume a triangle with sides $R_{E}$, $R_{E}+h_{2}(r, \alpha)$, and $r / 3$, and an angle opposite $\left(R_{E}+h_{2}(r, \alpha)\right)$ of $(\alpha+\pi / 2)$ in a similar way to the $\frac{1}{2}$-hop backscatter case. Hence, using the same method we can determine the virtual height as,

$h_{2}(r, \alpha)=\left(R_{E}^{2}+(r / 3)^{2}+2(r / 3) R_{E} \sin \alpha\right)^{\frac{1}{2}}-R_{E}$

In Fig. $4 \mathrm{~b}$ we present the real space distribution of virtual height determined using Eq. (3), in the same format as Fig. 4a. Here, only the distribution at far ranges corresponds to $1 \frac{1}{2}$-hop backscatter and so we will only discuss this distribution. The $1 \frac{1}{2}$-hop F-region backscatter extends over ground ranges of $\sim 1700-3300 \mathrm{~km}$ with the peak virtual height occurring at $\sim 300-400 \mathrm{~km}$. The ground range locations imply that the initial reflection on the virtual propagation path occurs at $\frac{1}{2}$-hop distances of $\sim 560-1100 \mathrm{~km}$ which corresponds to the lower ranges of the $\frac{1}{2}$-hop F-region distribution in Fig. 4a where the virtual height distribution peaks around $\sim 400 \mathrm{~km}$.

2.4 Uncertainties in mapping when using the standard SuperDARN virtual height model

We can use our measured virtual height distribution to investigate the uncertainties in the estimation of virtual propagation paths, and their associated ground ranges, that are introduced by using the standard SuperDARN virtual height model. For our $\frac{1}{2}$-hop backscatter distribution we can assume the same triangle as in the previous section and determine the angle $\phi_{1}(r, \alpha)$ opposite $r$ as

$\phi_{1}(r, \alpha)=\sin ^{-1}\left[\frac{r \cos \alpha}{R_{E}+h_{1}(r, \alpha)}\right]$

The estimated ground range is the arc of the angle $\phi_{1}$ on the Earth's surface and hence,

$G_{1}(r, \alpha)=R_{E} \phi_{1}(r, \alpha)=R_{E} \sin ^{-1}\left[\frac{r \cos \alpha}{R_{E}+h_{1}(r, \alpha)}\right]$

Most SuperDARN data analysis uses the standard virtual height model presented in the Introduction when mapping the geographic/geomagnetic locations of the scattering point, and elevation angle data is either not available or not used. When using such a virtual height model, in which the virtual height $h^{*}(r)$ varies with range $r$ only, Eq. (5) can only be used to estimate the ground range by determining an "effective" elevation angle which is consistent with the range and virtual height. Alternatively, we can assume a triangle with sides $R_{E},\left(R_{E}+h^{*}(r)\right)$, and $r$, and an unknown angle $\phi^{*}(r)$ opposite $r$ to give

$\phi^{*}(r)=\cos ^{-1}\left[\frac{R_{E}^{2}+\left(R_{E}+h^{*}(r)\right)^{2}-r^{2}}{2 R_{E}\left(R_{E}+h^{*}(r)\right)}\right]$

where the model-determined ground range is the arc of the angle $\phi^{*}$ on the Earth's surface and can be written as,

$$
\begin{aligned}
G^{*}(r) & =R_{E} \phi^{*}(r) \\
& =R_{E} \cos ^{-1}\left[\frac{R_{E}^{2}+\left(R_{E}+h^{*}(r)\right)^{2}-r^{2}}{2 R_{E}\left(R_{E}+h^{*}(r)\right)}\right]
\end{aligned}
$$


We can use our statistical database to provide an estimate of the typical uncertainties in the ground range measurement (and hence, in the mapping of the scattering region) that result from using the standard SuperDARN virtual height model. In the top panel of Fig. 5 we present the distribution of measured virtual height with range for our Saskatoon beam 3 database as presented already in Fig. 4. In the first instance we will concentrate on $\frac{1}{2}$-hop E- and Fregion backscatter only which we have approximated to be extending from the lowest ranges to the dotted vertical line at $\sim 2150 \mathrm{~km}$ range (this is the range at which the peak in the range-elevation angle distribution in Fig. 3 changes from the $\frac{1}{2}$-hop to the $1 \frac{1}{2}$-hop distribution). The solid blue line shows the standard SuperDARN virtual height model as outlined in Eq. (1). For much of the E-region distribution and for the lower range F-region distribution the standard model matches quite well to the observed virtual height distribution, although the simplified model variation in the E- to F-region transition leads to differences. The largest differences, however, are in the far range $\frac{1}{2}$-hop F-region distribution ( $\sim 1500-2150 \mathrm{~km}$ range) where the peak of the observed virtual height distribution increases with range to $\sim 800 \mathrm{~km}$, twice the typical standard model value.

In the middle panel of Fig. 5 we present the distribution of the estimated ground range with range for our database (determined using Eq. 5). The solid blue line illustrates the estimated ground range when employing the standard SuperDARN virtual height model (determined using Eq. 7). At low ranges $(<\sim 1200 \mathrm{~km}$ range) the overlap between the two ground range estimations is very good, illustrating that the standard model is generally providing a good estimate of the ground range, and hence, the location of the scattering region. However, for the far range $\frac{1}{2}$-hop F-region backscatter the ground range determined using the standard virtual height model moves increasingly further from our measured ground range distribution.

In the bottom panel of Fig. 5 we show the difference between the standard model ground range and our measured ground range distribution, as determined from

$\varepsilon_{G^{*}}(r, \alpha)=G^{*}(r)-G_{1}(r, \alpha)$

The blue line at zero difference effectively marks our estimate of the scattering location. A positive difference indicates that the standard model places our scattering points farther from the radar than this, whereas a negative difference indicates that the standard model places our scattering points closer to the radar than this. This panel confirms that the main uncertainties in determining the $\frac{1}{2}$-hop backscatter location using the standard model occur in the E-region/Fregion overlap region, and at far ranges, as discussed above. At ranges of $\sim 2000 \mathrm{~km}$ the standard model places the scattering locations at $\sim 100-250 \mathrm{~km}$ farther away from the radars than the measured locations. For a meridional SuperDARN beam, this would represent $\sim 0.9-2.3^{\circ}$ of latitude.
Determining a similar estimation of the ground range difference for $1 \frac{1}{2}$-hop backscatter is more complex. The ground range of our measured distribution can be estimated using a similar method to that shown above, using the same triangle used to determine the $1 \frac{1}{2}$-hop backscatter virtual height distribution in the previous section, but in this case determining the angle $\phi_{2}(r, \alpha) / 3$ opposite $r / 3$, and using this to estimate the ground range as follows,

$G_{2}(r, \alpha)=R_{E} \phi_{2}(r, \alpha)=3 R_{E} \sin ^{-1}\left[\frac{(r / 3) \cos \alpha}{R_{E}+h_{2}(r, \alpha)}\right]$

This uses the true virtual height $h_{2}(r, \alpha)$ of this distribution as determined in the previous section. This virtual height distribution is shown in the far range section $(>\sim 2150 \mathrm{~km}$ range) in the top panel of Fig. 5 (marked "True"). This distribution matches closely to the standard virtual height model at these ranges. This is misleading, however, as the ground range estimated from our measured virtual height distribution assumes that the backscatter is all $1 \frac{1}{2}$-hop whereas the standard SuperDARN algorithm assumes that the backscatter is all $\frac{1}{2}$-hop. Hence, when we estimate the corresponding ground ranges and the ground range difference, as we did for the $\frac{1}{2}$-hop backscatter distribution, using in this case

$\varepsilon_{G^{*}}(r, \alpha)=G^{*}(r)-G_{2}(r, \alpha)$

we find that the ground range difference in this region is in fact quite significant, with the standard model once again placing the scattering regions at $\sim 100-300 \mathrm{~km}$ farther away from the radar than the measured locations (bottom panel of Fig. 5).

In order to develop a new SuperDARN virtual height model in which the virtual height varies solely with range, as in the present standard model, we need to retain the assumption of $\frac{1}{2}$-hop propagation at all ranges in order to interface with present SuperDARN software. To achieve this when dealing with backscatter that is truly $1 \frac{1}{2}$-hop we introduce the concept of a pseudo virtual height $h_{p}$. The pseudo virtual height is the virtual height that one needs to assume for $1 \frac{1}{2}$ hop backscatter in order to retain the correct ground range when using a $\frac{1}{2}$-hop backscatter assumption when mapping the location of the scattering region. The concept of pseudo virtual height is explained pictorially in Fig. 6. Figure 6 shows the propagation scenario for $1 \frac{1}{2}$-hop backscatter as presented previously in Fig. 1c. In addition we have added a $\frac{1}{2}$-hop pseudo virtual path in blue which has the same range as the actual $1 \frac{1}{2}$-hop virtual path shown in black, and which also has the same ground range. Consequently, the pseudo virtual scattering point is at a much greater altitude than the actual virtual scattering point, but gives the correct ground range when $\frac{1}{2}$-hop propagation is assumed.

To determine the pseudo virtual height we assume a triangle with sides $R_{E}, R_{E}+h_{p}(r, \alpha)$, and $r$, where the angle opposite $r$ is $\phi_{2}(r, \alpha)$. This is consistent with the measured ground range $G_{2}(r, \alpha)$. The pseudo virtual height can then 


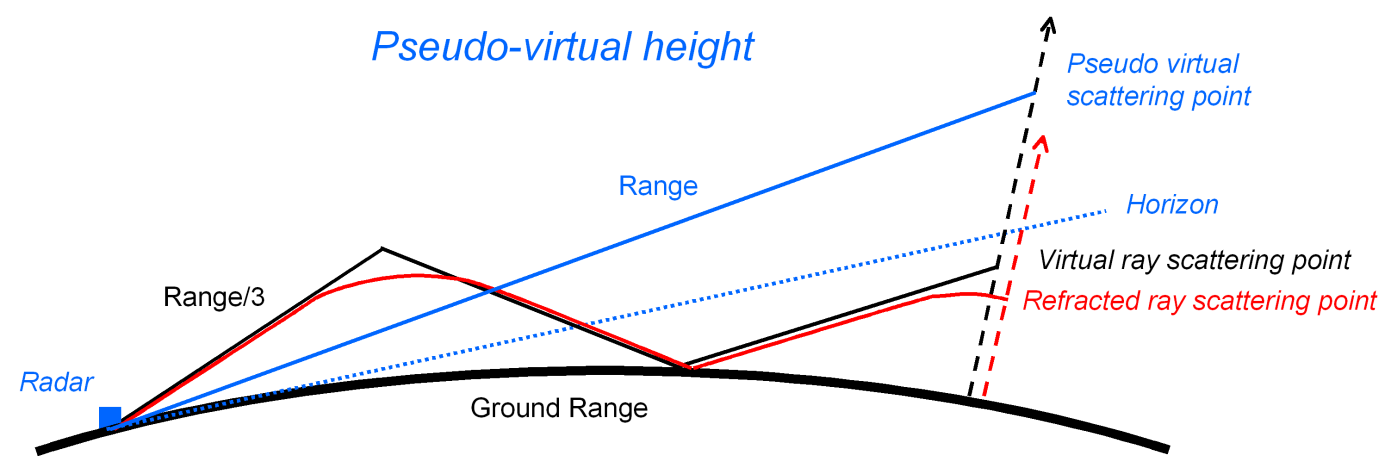

Fig. 6. A simple schematic diagram illustrating the concept of pseudo virtual height for $1 \frac{1}{2}$-hop backscatter. The curved bold black line represents the surface of the Earth in the plane of the propagation path, the solid black line represents a virtual propagation path, the solid red line represents a refracted propagation path, the dashed black and red lines are perpendicular to the Earth's surface, passing through the virtual and refracted ray scattering points, respectively, and the dotted blue line is the horizon. The solid blue line represents the pseudo virtual propagation path. (see text for full details).

be determined using simple trigonometry as,

$$
\begin{aligned}
h_{p}(r, \alpha)= & \left(r^{2}-R_{E}^{2} \sin ^{2} \phi_{2}(r, \alpha)\right)^{\frac{1}{2}} \\
& -R_{E}\left(1-\cos \phi_{2}(r, \alpha)\right)
\end{aligned}
$$

The top panel of Fig. 5 shows the pseudo virtual height distribution for our database (marked "Pseudo"). The distribution of pseudo virtual heights is at greater altitudes than the true virtual height distribution and stretches from $\sim 600$ $1000 \mathrm{~km}$, peaking at $\sim 800 \mathrm{~km}$.

2.5 Uncertainties in mapping when using the peak virtual height variation

It is possible to remove much of the systematic uncertainty from the ground range difference distribution shown in Fig. 5 by using the peak virtual height of the Saskatoon beam 3 database as a simple virtual height model. The peak virtual height variation is determined by taking the peak occurrence value in a smoothed version of the virtual height distribution at every range. The top panel in Fig. 7 presents the Saskatoon beam 3 virtual height distribution as presented already in the top panel of Fig. 5. However, now the blue line represents the variation of the peak occurrence value of the distribution with range. In the $1 \frac{1}{2}$-hop propagation region this is the variation of the pseudo virtual height distribution (this allows us to use the $\frac{1}{2}$-hop backscatter assumption at all ranges when estimating the location of the scattering region). Surprisingly, the transition of this peak value from the $\frac{1}{2}$-hop to $1 \frac{1}{2}$-hop backscatter region is not characterised by a large discontinuity and there is only a small transition between the two regions. However, understanding this overlap region is complicated as will be discussed in full detail later in the paper.

We can use the peak occurrence value (blue line) as a basic empirical virtual height model to see how it improves on the standard SuperDARN virtual height model. As would be expected, given its origin, using the peak virtual height variation to estimate our ground range and the associated ground range differences (as we did for the standard model in Fig. 5), largely removes the systematic uncertainties that exist when using the standard model. This is shown clearly in the lower two panels in Fig. 7. In the bottom panel, the blue line at zero difference once again represents the measured ground range. A positive difference indicates that using the peak virtual height variation is placing our scattering points farther from the radar than this, whereas a negative difference indicates that it is placing our scattering points closer to the radar than this. This figure clearly shows that the ground range differences for our distribution are now approximately symmetric around the measured ground range value, with most of the distribution being contained within $\sim 100 \mathrm{~km}$ of the measured ground range value. Hence, using this simple empirical virtual height model has removed the major systematic uncertainties in the ground range determination that were clearly apparent in Fig. 5, leaving predominantly random uncertainties resulting from a combination of diurnal, seasonal and solar cycle factors, signal frequency variations, and general spatial and temporal ionospheric density variability. This peak virtual height variation provides the basis for our determination of a new empirical virtual height model.

\subsection{Dependence on signal frequency}

As discussed above, the propagation paths of HF signals are highly dependent on the frequency of the signal, with higher frequency signals refracting less than lower frequency signals. Additionally, the peak elevation angle of the vertical radiation pattern will change with frequency. Hence, changing the signal frequency will change the most likely propagation paths and hence, change the range-elevation angle distribution shown in Fig. 3. Hence, our peak virtual height 


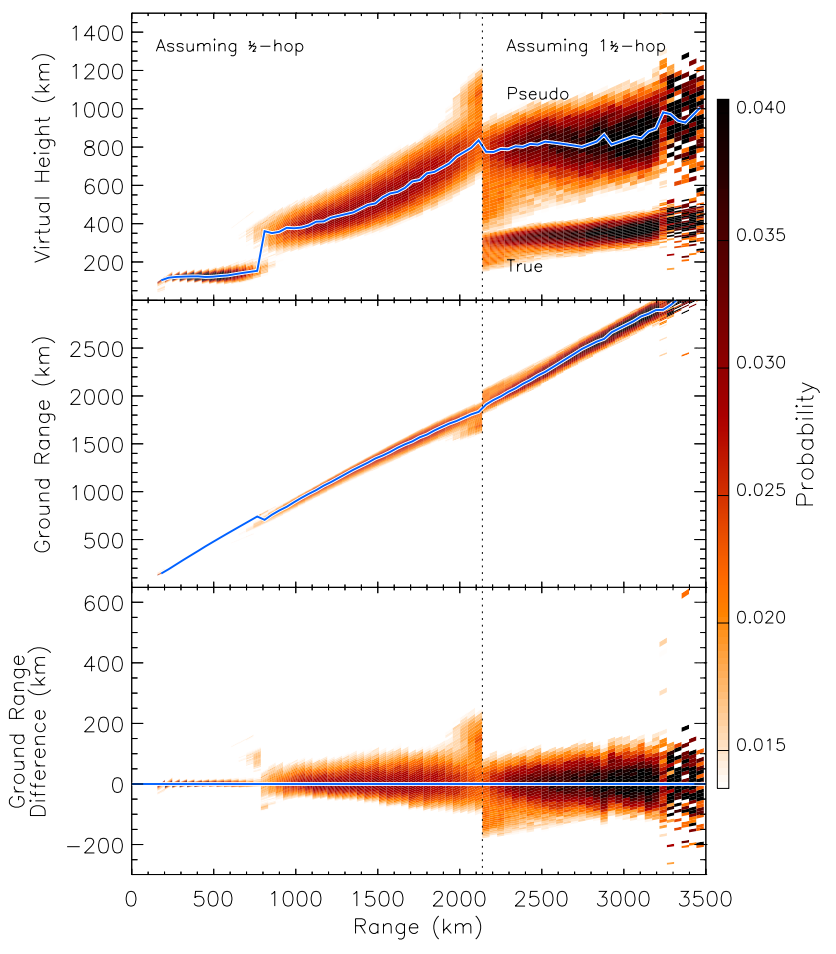

Fig. 7. A comparison of the variation of virtual height and ground range with range between the measured Saskatoon beam 3 backscatter distribution (colour contours) and the peak virtual height variation derived from the beam 3 data set (blue line). (top) The virtual height distribution of Saskatoon beam 3 backscatter from 19972001 inclusive (adapted from Fig. 4). $\frac{1}{2}$-hop backscatter is assumed up to a range of $2135 \mathrm{~km}$ (vertical dotted line), whereas $1 \frac{1}{2}$-hop backscatter is assumed at higher ranges. At higher ranges two distributions (true and pseudo) are shown, as in Fig. 5. (middle) The ground range distribution for the same data set. (bottom) The ground range difference distribution showing the range of errors that result from using the peak virtual height variation as a simple empirical virtual height model. Here, the blue line represents the measured distribution and the contours represent the differences that occur from using the peak virtual height variation. (all panels) Only common mode backscatter with a line-of-sight velocity magnitude greater than $100 \mathrm{~m} / \mathrm{s}$ are included. The distribution in each range bin $(45 \mathrm{~km})$ has been normalised. The colour scale provides a key to the occurrence probability in each region; occurrence probabilities of less than 0.014 are shown as white space.

profile will vary with signal frequency. Typically, the signal frequencies used by a particular radar are varied to provide the best HF propagation paths for achieving orthogonality with the magnetic field over the widest number of ranges at a particular time. Hence, different frequencies are often used for dayside and nightside ionospheric propagation (when the ionospheric electron density profiles are very different), and for winter and summer ionospheric propagation (for the same reason).
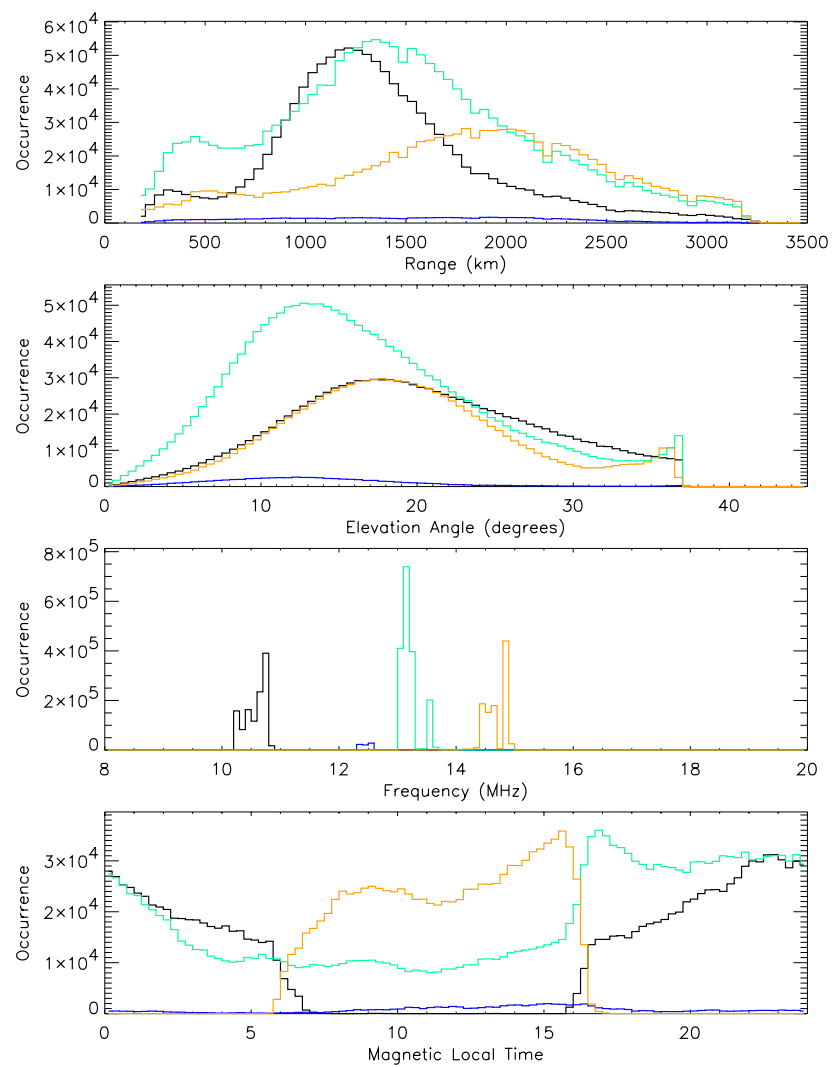

Fig. 8. Statistical histograms of range, elevation angle, frequency, and magnetic local time for Saskatoon beam 3 common mode data from 1997-2001 inclusive, separated into 4 different frequency bands. The black, blue, green, and yellow curves show the histograms for frequency ranges $10-11 \mathrm{MHz}, 12-13 \mathrm{MHz}, 13$ $14 \mathrm{MHz}$, and 14-15 MHz, respectively.

In this study we have separated our Saskatoon beam 3 distribution into four separate histograms based on the signal frequency: $10-11 \mathrm{MHz}, 12-13 \mathrm{MHz}, 13-14 \mathrm{MHz}$, and 14 $15 \mathrm{MHz}$. In Fig. 8 we present the statistical histograms of range, elevation angle, frequency, and MLT for the data in these four frequency ranges in the same format as Fig. 2. Here, the black, blue, green, and orange histograms represent data with signal frequencies of $10-11 \mathrm{MHz}, 12-13 \mathrm{MHz}$, $13-14 \mathrm{MHz}$, and $14-15 \mathrm{MHz}$, respectively. Figure 8 shows that changing the signal frequency changes the ranges of the most-likely scattering regions; the peaks in the range occurrence distributions (top panel) are up to $800 \mathrm{~km}$ apart. The elevation angle histograms are also changed with the peaks in occurrence (second panel) being $\sim 4.5^{\circ}$ apart. However, as the MLT histogram in the bottom panel shows, it is difficult to compare these histograms and interprete the differences as wholly due to changes in signal frequency because of the significantly different MLT distributions at different frequencies. Also, there are significant seasonal differences which are not shown as part of this figure. Hence, differences 


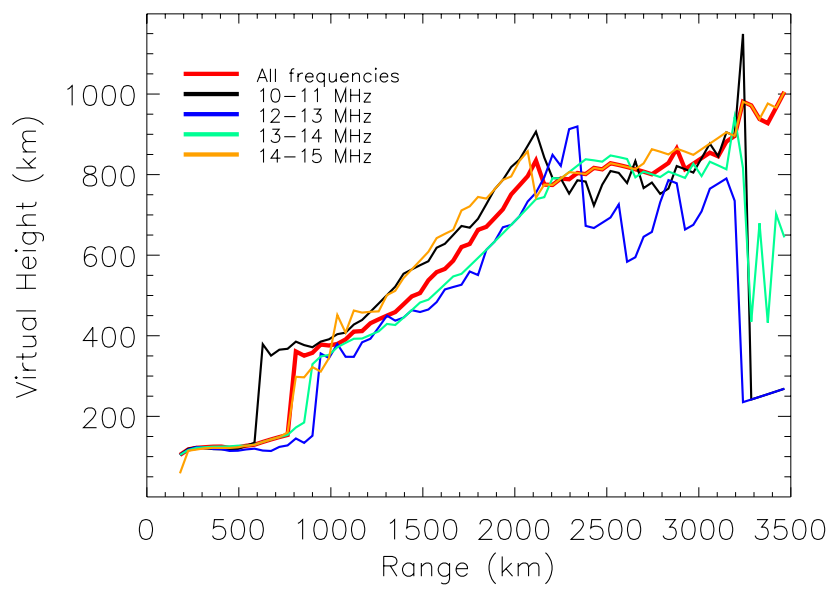

Fig. 9. Peak virtual height variations determined for each frequency range. The black, blue, green, and yellow curves show the variations for frequency ranges $10-11 \mathrm{MHz}, 12-13 \mathrm{MHz}, 13-14 \mathrm{MHz}$, and $14-15 \mathrm{MHz}$, respectively. The red curve shows the peak virtual height variation determined when using the whole data set (all frequencies) as shown in Fig. 7.

between the four different histograms are not due to changes in the signal frequency alone but to a combination of frequency differences, MLT differences, and seasonal differences.

In order to study the differences between the virtual height distributions in the four frequency bands we have determined the variation with range of the peak virtual height value for each band, as in Fig. 7. In Fig. 9 we present these peak virtual height variations along with that for all frequencies (red curve), as was presented in Fig. 7. The peak virtual height variations are similar, although poor statistics at far ranges for some frequencies has resulted in larger variations away from the "all frequencies" curve. The transition between the dominance of E- and F-region backscatter also occurs at different ranges for the different frequency bands. For most ranges all the virtual height curves are within $\sim 50 \mathrm{~km}$ of the "all frequencies" curve.

Because of the complex issue of the combined effects of frequency, MLT and season, we make the decision at this time not to determine separate virtual height models for different frequencies, and will determine a single virtual height model based on the "all frequencies" curve which can be used for all frequencies, times, and conditions. In the future it may be more practical to determine different virtual height models which are better suited to different frequencies, MLTs, seasons, etc.

\subsection{Dependence on beam direction}

Until now, our analysis has involved solely Saskatoon beam 3 data. Saskatoon beam 3 points approximately in the geomagnetic meridional direction. As different beams of the Super-
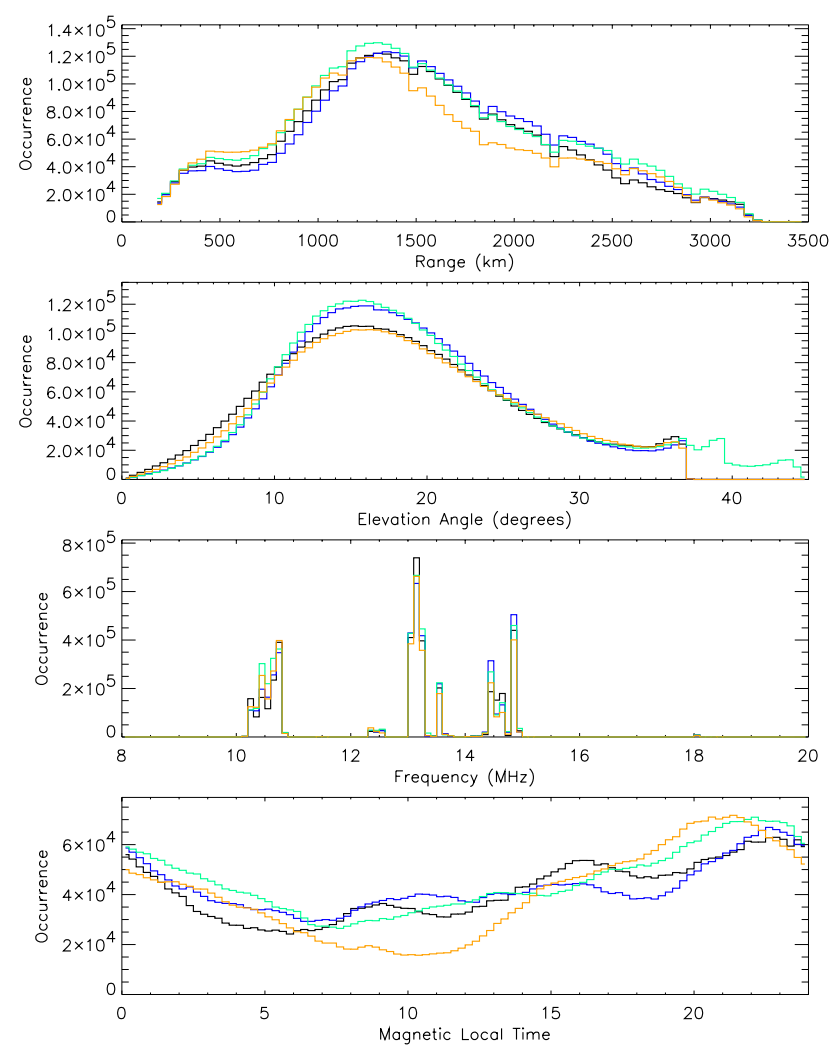

Fig. 10. Statistical histograms of range, elevation angle, frequency, and magnetic local time for common mode data from four different Saskatoon beams from 1997-2001 inclusive. The black, blue, green, and yellow curves show the histograms for beams 3, 6, 9, and 12 , respectively.

DARN radars sample the ionosphere over different latitudinal ranges they consequently encounter a range of different ionospheric conditions. The standard 16 beams which cover the field of view of each SuperDARN radar will also encounter the Earth's magnetic field at a range of angles and so different amounts of refraction will be necessary to achieve orthogonality with the magnetic field. Hence, we need to test if these differences have a major effect on the average HF propagation and hence on the virtual height distributions with range.

In Fig. 10 we present the statistical histograms of range, elevation angle, frequency, and MLT for the data from four Saskatoon beams, in the same format as Fig. 2. Here, the black, blue, green, and orange histograms represent data from beams $3,6,9$, and 12 , respectively, which are directed approximately $0^{\circ}, 10^{\circ}, 20^{\circ}$, and $30^{\circ}$ away from the meridional direction, respectively. Figure 10 shows that the statistical distributions for these different beams are extremely similar, the only significant differences being apparent in the MLT histograms. Similarly, when we plot the peak virtual height variations for these four beams in Fig. 11 (as we did for the different frequencies in Fig. 9), it is clear that these 


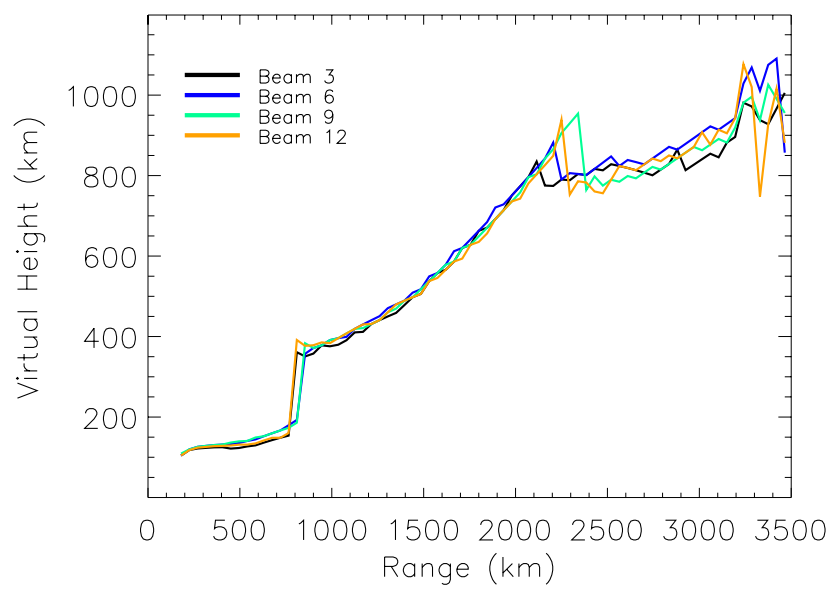

Fig. 11. Peak virtual height variations determined for four different beams. The black, blue, green, and yellow curves show the variations for beams $3,6,9$, and 12 , respectively.

virtual height variations are almost identical. Therefore, we can say that the radar beam direction (within the range of beam orientations that we have investigated) has very little influence on the peak virtual height variation with range. The only difference appears to be the range at which the dominance of the distribution switches from $\frac{1}{2}$-hop to $1 \frac{1}{2}$-hop backscatter which varies by $\sim 200 \mathrm{~km}$.

\section{A new empirical virtual height model}

\subsection{Model determination}

We choose to base our new empirical virtual height model on the peak virtual height variations presented in Fig. 11. In Fig. 12 we present these virtual height variations again (as orange lines). The dashed vertical lines indicate where we have previously delineated between $\frac{1}{2}$-hop E-region, $\frac{1}{2}$ hop F-region, and $1 \frac{1}{2}$-hop F-region backscatter, based on the Saskatoon beam 3 database. It is clear from these virtual height variations that there are three distinct regions of the curve which require separate models. In each of the three backscatter regions we have fitted a low-order polynomial (quadratic) of the form

$h^{*}(r)=A+B r+C r^{2}$

to the average virtual height curve determined from the peak virtual height variations measured on the four Saskatoon beams. The coefficients of the fits (A, B, and C) are presented in Table 1. The fitted curves are also shown in Fig. 12: the green curve represents the $\frac{1}{2}$-hop E-region fit, the black curve represents the $\frac{1}{2}$-hop F-region fit, and the red curve represents the $1 \frac{1}{2}$-hop F-region fit. The fits in all regions are extremely good, providing an excellent representation of the average peak virtual height variation. The quadratic form of the fits

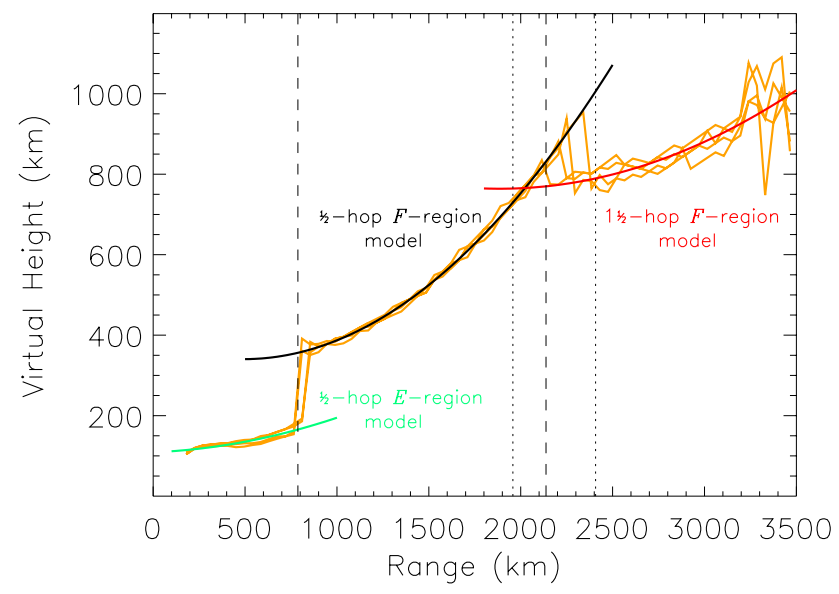

Fig. 12. The virtual height models determined from the Saskatoon data set. The yellow lines represent the peak virtual height variations determined for four different beams as shown in Fig. 11. The green curve shows the $\frac{1}{2}$-hop E-region model determined by fitting a quadratic to the data below $790 \mathrm{~km}$ range (as highlighted by the vertical dashed line). The black curve shows the $\frac{1}{2}$-hop Fregion model determined by fitting a quadratic to the data between 790 and $2130 \mathrm{~km}$ range (as highlighted by the two vertical dashed lines). The red curve shows the $1 \frac{1}{2}$-hop F-region model determined by fitting a quadratic to the data above $2130 \mathrm{~km}$ range. The two vertical dotted lines delineate the $\frac{1}{2}$-hop and $1 \frac{1}{2}$-hop F-region overlap region where backscatter for both propagation modes occurs with a significant probability.

Table 1. The coefficients for Eq. (12) for the three different virtual height models.

\begin{tabular}{lccc}
\hline Backscatter Type & A & B & C \\
\hline$\frac{1}{2}$-hop E-region & 108.974 & 0.0191271 & $6.68283 \times 10^{-5}$ \\
$\frac{1}{2}$-hop F-region & 384.416 & -0.178640 & $1.81405 \times 10^{-4}$ \\
$1 \frac{1}{2}$-hop F-region & 1098.28 & -0.354557 & $9.39961 \times 10^{-5}$ \\
\hline
\end{tabular}

also allows easy integration with existing SuperDARN algorithms and software.

\subsection{Ground range difference distributions}

In order to evaluate to what extent our new model virtual heights are an improvement on those of the standard SuperDARN model we can compare in detail the ground range difference distributions obtained when using the different models in each of the three backscatter regions for the Saskatoon beam 3 data set. Figure 13 presents the distributions of ground range difference in all these cases. The dotted vertical line at zero difference represents the estimated ground range location $\left(G_{1}\right.$ or $\left.G_{2}\right)$ determined using the measured range and elevation angles. The red curves show the distributions of ground range difference when using the standard 


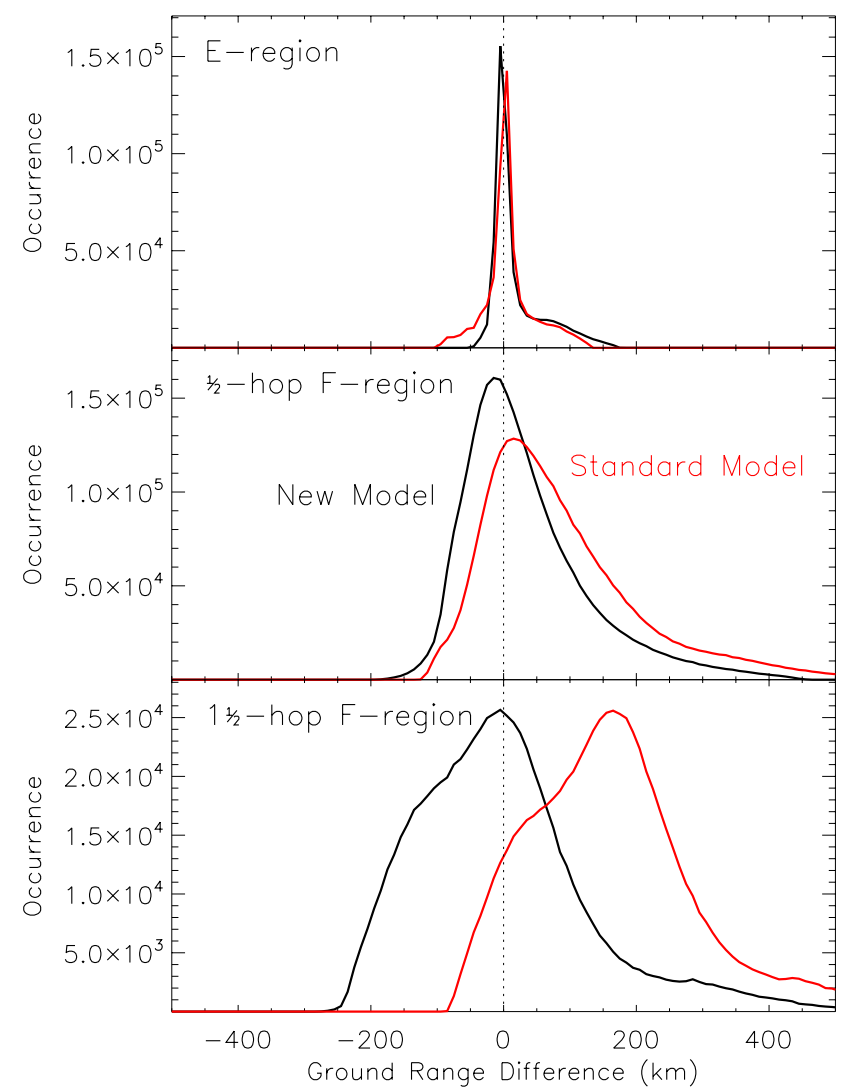

Fig. 13. Ground range difference distributions comparing the performance of the new empirical model (black distributions) and the standard SuperDARN model (red distributions). The distributions are determined by comparing all the measured ground range values from the Saskatoon beam 3 data set with the ground range values predicted by the models. The distributions for the $\frac{1}{2}$-hop E-region models use data below $790 \mathrm{~km}$ range. The distributions for the $\frac{1}{2}$ hop F-region models use data between 790 and $2130 \mathrm{~km}$ range. The distributions for the $1 \frac{1}{2}$-hop F-region models use data above $2130 \mathrm{~km}$ range.

SuperDARN virtual height model. The black curves show the distributions of ground range difference when using our new empirical virtual height models.

The top panel of Fig. 13 presents the ground range differences for the two models in the $\frac{1}{2}$-hop E-region. Here, both models perform extremely well, with most of the ground range difference estimates being less than $\sim 25 \mathrm{~km}$. Most of the larger differences here arise as a result of the Eto F-region transition, i.e. some of the low range F-region backscatter is being treated as E-region backscatter here.

The middle panel of Fig. 13 presents the ground range differences for the two models in the $\frac{1}{2}$-hop F-region. One immediate observation is that the width of the difference distributions for this region has greatly increased in comparison to the E-region distributions. This increased spread of the distributions is due to a combination of factors, predominantly the increase in the natural variability in the ionospheric electron density (and hence the HF propagation paths) over a longer propagation path. Errors in the determination of the elevation angle will also play a role, especially for backscatter at far ranges. Our model, based on the most likely propagation paths, will only remove systematic errors from the ground range estimations. A reduction of the random errors which lead to the spread in the distribution might be reduced by the use of multiple virtual height models matching all signal frequencies, and all MLT and seasonal conditions. Even then, much of the spread of the distributions may remain due to the natural ionospheric variability and elevation angle errors. The distribution of ground range differences from using the standard model (red) is also highly skewed to positive ground range differences suggesting that it typically places the scattering region farther from the radar than its true location. However, the peak difference value from this model is still close to zero showing that in many cases it provides a fair approximation of the ground range. Using our new empirical model (black) reduces the skew of the ground range difference distribution and moves the centre of the distribution closer to zero ground range difference.

The bottom panel of Fig. 13 presents the ground range differences for the two models in the $1 \frac{1}{2}$-hop F-region. Here, the width of the distributions has increased, further highlighting the increased uncertainty about the longer propagation paths associated with $1 \frac{1}{2}$-hop backscatter. Here, both the ground range difference distributions are very similar in shape. However, the difference distribution for our new model (black) is centred close to zero ground range difference whereas that for the standard model (red) is centred close to $\sim 150 \mathrm{~km}$. This suggests that the standard model is typically placing $1 \frac{1}{2}$ hop F-region backscatter at a distance of $\sim 150 \mathrm{~km}$ farther away from the radar than the true backscattering location. The hump on the left-hand side of both these distributions is a result of the overlap region between $\frac{1}{2}$-hop and $1 \frac{1}{2}$-hop F-region backscatter and exaggerates the spread of the distributions.

In Fig. 14 we study the F-region ground range difference distributions in more detail, concentrating on the distributions at single range gates from range gate $20(1035 \mathrm{~km}$ range), every 5 range gates to range gate $65(3060 \mathrm{~km})$. At range gate $20(1035 \mathrm{~km})$ the two models have very similar virtual heights giving almost identical ground range difference distributions which are relatively narrow. As the range increases within the $\frac{1}{2}$-hop F-region $(<\sim 2150 \mathrm{~km})$, the difference distributions become increasingly wider. Whereas the peak of our new model distribution (black) stays close to zero, the peak of the standard model distribution (red) moves increasingly further from zero, being $\sim 120 \mathrm{~km}$ at range gate $40(1935 \mathrm{~km})$. At range gate $45(2160 \mathrm{~km})$ the difference distributions are clearly double peaked. This is because these distributions contain a mixture of $\frac{1}{2}$-hop and $1 \frac{1}{2}$-hop F-region backscatter. The left hand peak represents 
the $\frac{1}{2}$-hop backscatter peak whereas the right hand peak represents the $1 \frac{1}{2}$-hop backscatter peak. Hence, the difference distributions for range gate 45 are somewhat misleading. For this range, the measured ground range locations have been determined assuming that the backscatter is all $1 \frac{1}{2}$-hop backscatter, and so we should only compare the right-hand peaks of the distributions with the zero ground range difference line. Consequently, the right-hand peak of our new model distribution is located close to zero ground range difference whereas the right-hand peak of the standard model distribution is located at $\sim 150 \mathrm{~km}$. At range gate 50 $(2385 \mathrm{~km})$ the ground range difference distributions are still double peaked, but here, the $1 \frac{1}{2}$-hop F-region backscatter peak is becoming more dominant over the $\frac{1}{2}$-hop backscatter peak. Here again, the right-hand peak of our new model distribution is located close to zero ground range difference whereas the right-hand peak of the standard model distribution is located at $\sim 150 \mathrm{~km}$. At range gate $55(2610 \mathrm{~km})$, and at farther ranges, the $\frac{1}{2}$-hop backscatter peak has almost disappeared and only the $1 \frac{1}{2}$-hop backscatter peak remains. At these ranges the peak of our new model distribution is still located close to zero ground range difference whereas the peak of the standard model distribution is located $\sim 150-200 \mathrm{~km}$ farther away from the radar.

3.3 Detailed analysis of the $\frac{1}{2}$-hop to $1 \frac{1}{2}$-hop backscatter overlap region

A number of the figures in this paper have shown that there are a selection of range gates for which both $\frac{1}{2}$-hop and $1 \frac{1}{2}$ hop F-region backscatter both exist in significant amounts. However, when mapping the location of our scattering region, we can only assume one of the two propagation modes in this region. If we are sure that the backscatter is of one or other type then there is no problem. However, if we are unsure as to whether the backscatter is $\frac{1}{2}$-hop or $1 \frac{1}{2}$-hop then we may be introducing error into our ground range determinations if we employ the wrong model. In this section we look in detail at the overlap region to investigate the errors that would be introduced by using the wrong virtual height model to estimate the location of the scattering region.

In Fig. 15 we show the ground range difference distributions from range gates 41 to 50 (ranges 1980 to $2385 \mathrm{~km}$ shown as dashed lines in Fig. 12) which result from using both our $\frac{1}{2}$-hop F-region virtual height model (blue) and our $1 \frac{1}{2}$-hop F-region virtual height model (yellow) assuming the backscatter is truly $\frac{1}{2}$-hop (hence the estimated ground range values are calculated assuming $\frac{1}{2}$-hop propagation). Hence, in this case we want the left-hand peak of our double-peaked distribution to be located at zero ground range difference. This left-hand peak is dominant at the lower ranges (which implies that most of the backscatter at these ranges is $\frac{1}{2}$ hop), but the $1 \frac{1}{2}$-hop backscatter peak becomes larger with increasing range until the two peaks are equal at $\sim 2160 \mathrm{~km}$

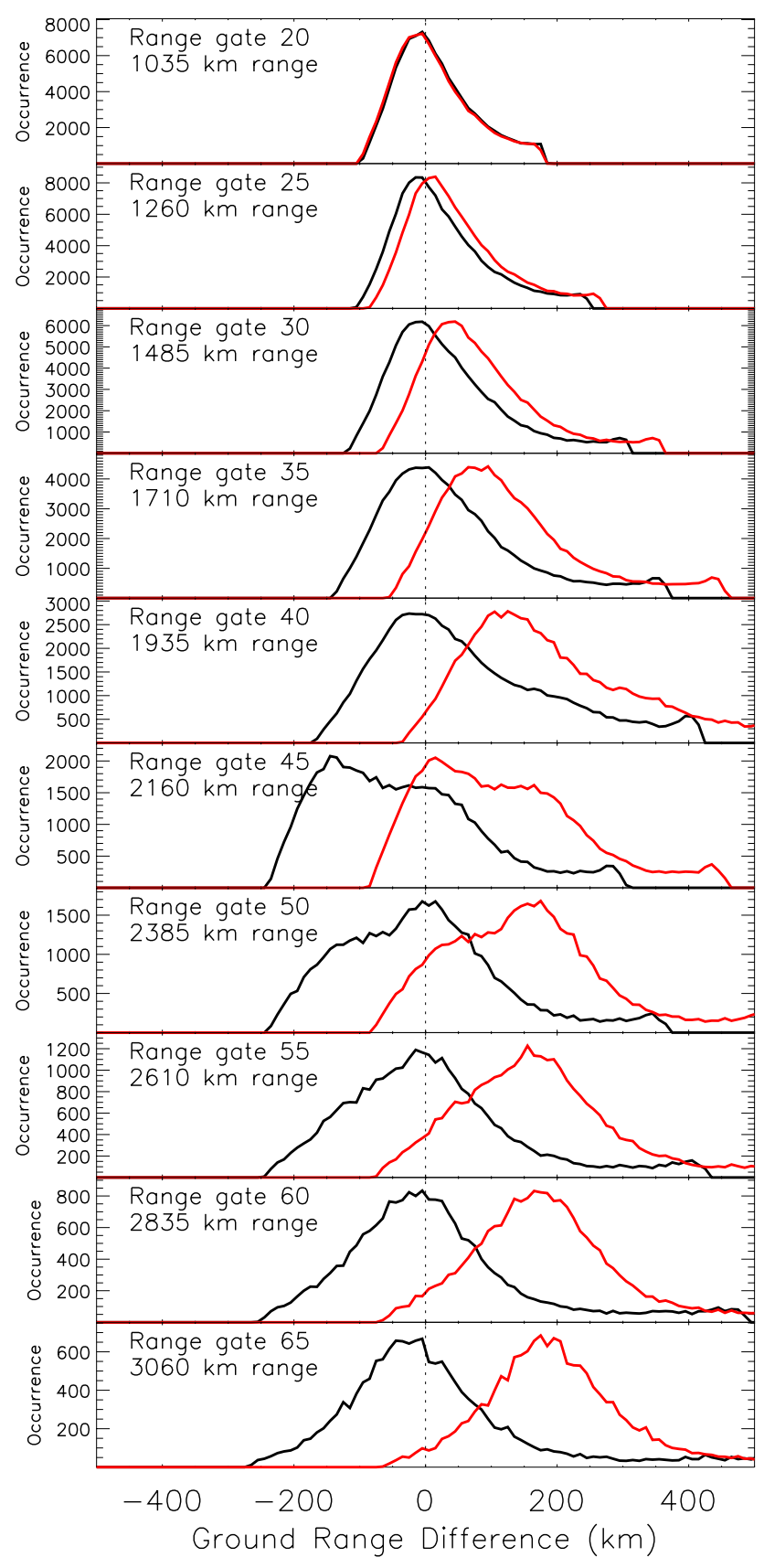

Fig. 14. Ground range difference distributions comparing the performance of the new empirical model (black distributions) and the standard SuperDARN model (red distributions) at a sample of single range gates. The distributions are determined by comparing all the ground range values measured at the specific range gate in the Saskatoon beam 3 data set with the ground range values predicted by the models. The ten panels show distributions every 5 range gates ranging from range gate $20(1035 \mathrm{~km}$ range; top panel) to range gate 65 (3060 km range; bottom panel). 


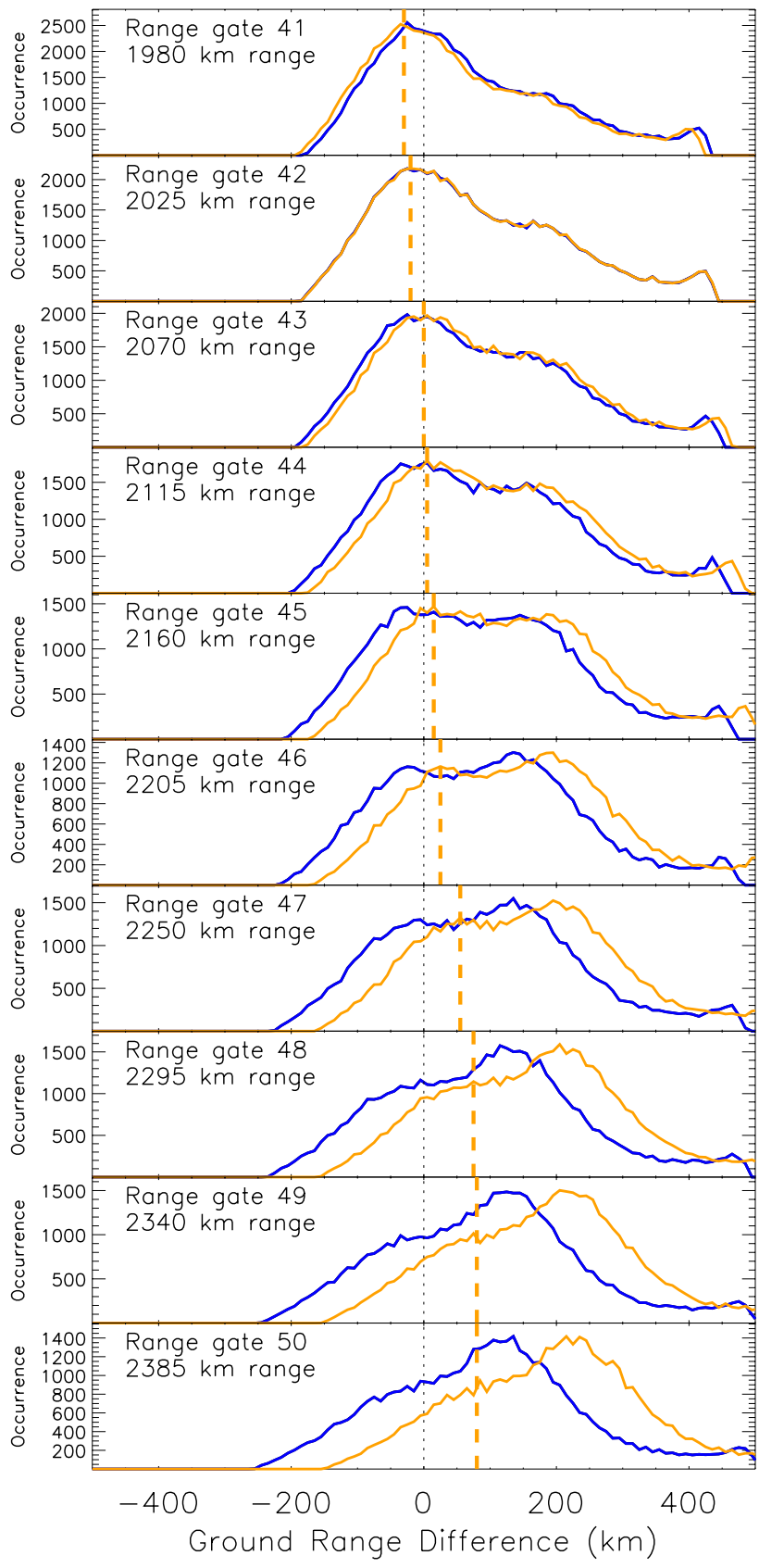

Fig. 15. Ground range difference distributions in the $\frac{1}{2}$-hop and $1 \frac{1}{2}$-hop F-region overlap region comparing the performance of the $\frac{1}{2}$-hop F-region model (blue distributions) and the $1 \frac{1}{2}$-hop F-region model (yellow distributions) when the measured backscatter is assumed to be truly $\frac{1}{2}$-hop. The distributions are shown at every range gate from range gate 41 (1980 $\mathrm{km}$ range; top panel) to range gate 50 ( $2385 \mathrm{~km}$ range; bottom panel). The vertical dashed yellow lines highlight the $\frac{1}{2}$-hop backscatter peak of the yellow distributions.

range, after which the $\frac{1}{2}$-hop peak starts to disappear, being largely reduced by $\sim 2385 \mathrm{~km}$ range. When using our $\frac{1}{2}$-hop F-region model (blue distribution) the left-hand peak of the distribution always occurs within $50 \mathrm{~km}$ of zero ground range difference, confirming the accuracy of the model. At the lower end of these 10 range gates $(\sim 1980-2205 \mathrm{~km}$ range), the left-hand peak of the difference distribution which results from using our $1 \frac{1}{2}$-hop F-region model (indicated by the dashed vertical yellow line on the yellow distribution) is also within $\sim 50 \mathrm{~km}$ of zero ground range difference. This is because the virtual heights for the two models in this region are very similar, as shown in Fig. 12. For greater ranges $(>2205 \mathrm{~km})$ the ground range difference of this left-hand peak increases. However, at the furthest range in Fig. 15 $(2385 \mathrm{~km})$, when the $\frac{1}{2}$-hop peak has nearly disappeared, the ground range difference of the left-hand peak is still less than $100 \mathrm{~km}$.

In Fig. 16 we show the ground range difference distributions for the same 10 range gates, but which result from using both our $\frac{1}{2}$-hop F-region virtual height model (blue) and our $1 \frac{1}{2}$-hop F-region virtual height model (yellow) assuming the backscatter is truly $1 \frac{1}{2}$-hop (hence the estimated ground range values are calculated assuming $1 \frac{1}{2}$-hop propagation). Hence, in this case we want the right-hand peak of our double-peaked distribution to be located at zero ground range difference. This right-hand peak is very small at lower ranges (implying that most of the backscatter at these ranges is $\frac{1}{2}$ hop as discussed above), but becomes larger as the range increases, finally dominating the data set at the furthest ranges being considered. When using our $1 \frac{1}{2}$-hop F-region model (yellow distribution) the right-hand peak of the distribution always occurs within $50 \mathrm{~km}$ of zero ground range difference, confirming the accuracy of the model. As with Fig. 15, because of the similarity of the virtual heights for the two models at the lower end of these 10 range gates $(\sim 1980-2160 \mathrm{~km}$ range), the right-hand peak of the difference distribution that results from using our $\frac{1}{2}$-hop F-region model (indicated by the dashed vertical blue line on the blue distribution) is also within $\sim 50 \mathrm{~km}$ of zero ground range difference. For greater ranges $(>2160 \mathrm{~km})$ the ground range difference of the righthand peak increases, reaching $\sim 120 \mathrm{~km}$ at $2385 \mathrm{~km}$ range.

From this analysis we can conclude that using the wrong virtual height model in the lower range portion of the overlap region will not lead to a significant increase in the ground range difference. However, for the higher ranges in this overlap region the errors become more significant (up to $\sim 120 \mathrm{~km}$ ). Our analysis shows that $\frac{1}{2}$-hop backscatter still occurs at these ranges because of propagation on Pedersen ray paths. Although the amount of $\frac{1}{2}$-hop backscatter is reduced at these ranges it is still significant enough that we cannot simply assume that all backscatter in this region is $1 \frac{1}{2}$-hop. Therefore, ground range uncertainties in this region are likely to be larger due to the possibility of the misidentification of the propagation mode. 


\section{Discussion}

We have shown in this paper that the development of a new virtual height model for SuperDARN which is based on measured elevation angle data significantly improves the accuracy of estimations of virtual ray propagation paths, and ultimately of the mapping of scattering locations, especially at far ranges. In this paper we have taken a simple approach and developed a single virtual height model which produces the best results for average propagation conditions, and hence reduces the systematic errors that occur when using the standard SuperDARN virtual height model. As is always the case when developing simple models, and in light of the remaining uncertainties that still exist when mapping scattering locations, it is important to consider how this model can be improved in the future. This requires a fuller understanding of the factors which are responsible for these uncertainties.

As discussed in the Introduction, one of the major uncertainties regarding the mapping of scattering regions is the offset that exists between the ground range of the virtual and refracted ray scattering points. This is presently a difficult problem to address, although ray-tracing studies using realistic ionospheric electron density profiles help in improving understanding of this problem. Other uncertainties in the mapping of the scattering region result from the natural variability of the ionospheric electron density which result in random uncertainties in the determination of real and virtual propagation paths. The minute-to-minute and hour-tohour temporal variability of the electron density, and its small and meso-scale spatial variability, which result from spatiotemporal magnetospheric, ionospheric, and atmospheric phenomena are factors that we cannot account for without making detailed spatiotemporal measurements of the electron density (which is not practical over large regions and over long timescales). Hence, the only part of the random uncertainties we can address is that associated with known changes in the average behaviour of the electron density. These effects are:

- Diurnal variations. The peak F-region electron density is typically much greater in the daytime ionosphere than in the nighttime ionosphere. Grouping elevation angle distributions according to MLT would allow different virtual height models to be determined for different times of day.

- Seasonal variations. The peak F-region electron density is typically greater in the winter hemisphere than in the summer hemisphere. Grouping elevation angle distributions according to season would allow different virtual height models to be determined for different seasons.

- Solar cycle variations. The peak F-region electron density is typically greater at times of solar maximum than at times of solar minimum. The altitude of the Fregion peak is also much higher on average at solar

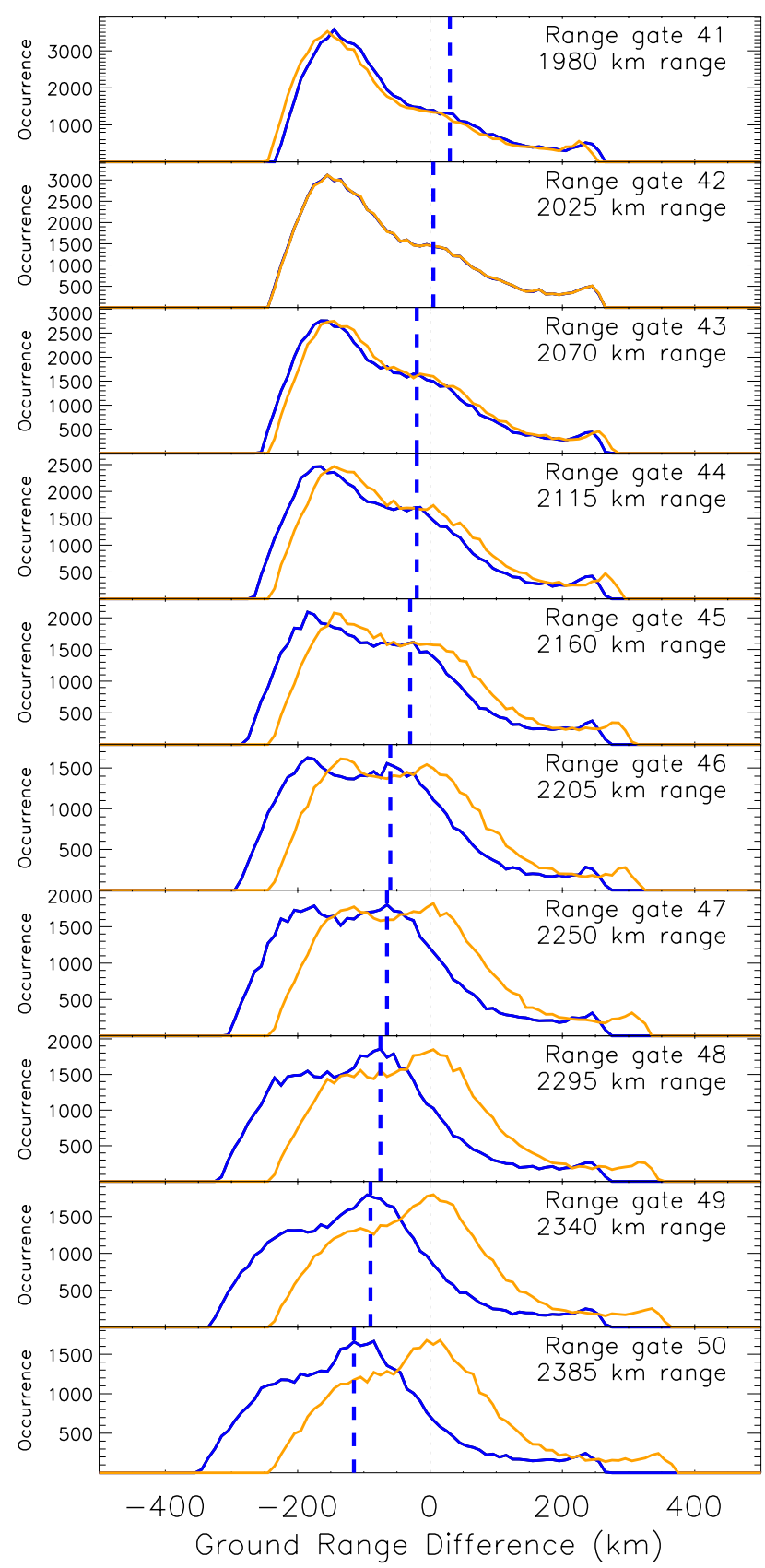

Fig. 16. Ground range difference distributions in the $\frac{1}{2}$-hop and $1 \frac{1}{2}$-hop F-region overlap region comparing the performance of the $\frac{1}{2}$-hop F-region model (blue distributions) and the $1 \frac{1}{2}$-hop F-region model (yellow distributions) when the measured backscatter is assumed to be truly $1 \frac{1}{2}$-hop. The distributions are shown at every range gate from range gate 41 (1980 $\mathrm{km}$ range; top panel) to range gate 50 (2385 km range; bottom panel). The vertical dashed blue lines highlight the $1 \frac{1}{2}$-hop backscatter peak of the blue distributions. 
maximum. Grouping elevation angle distributions according to their position in the solar cycle would allow different virtual height models to be determined for all parts of the solar cycle.

In addition to these three factors regarding the change in electron density with time there are two other factors which affect propagation that could be taken into account in the development of virtual height models. The first is the signal frequency, as discussed in detail earlier in the paper. To take this factor into account elevation angle distributions should also be grouped according to the operating frequency. The second factor, also discussed earlier in the paper, is the range of different ionospheric density profiles seen on average by the different radar beams. Although for the range of beams we studied in this paper, there was little variation in the peak virtual height between beams. However, it is not clear that this would be the case for all beams on all radars.

It is clear from the above discussion that there are a number of factors that affect propagation that could be taken into account when developing models of virtual height to be used with SuperDARN data. All of these factors need to be considered if our approach is to be taken forward and the model presented here is to be improved further. However, taking into account all these factors when developing virtual height models would lead to many additional complications. First, producing a statistically reliable virtual height model requires a large amount of elevation angle data. Depending on how the data sets were divided it is possible that for many combinations of conditions the statistics would not be sufficient to produce a reliable model, even if data from all radars and beam directions were combined. Hence, if these factors were to be taken into account it would be important not to overly categorise the data.

An alternative approach to increasing the accuracy of the mapping of scattering locations would be to make better use of the elevation angle data. As discussed in the Introduction the elevation angle allows an accurate determination of the virtual propagation path and allows for the possibility of dispensing with a virtual height model altogether. However, the elevation angles measured by a number of SuperDARN radars are often unreliable. In addition, it is not always possible to make elevation angle estimations for all backscatter measurements. Hence, for this approach to become a reality the reliability and accuracy of elevation angle measurements at all radars would need to be increased. The companion paper (Yeoman et al., 2008) discusses how the accuracy of ground range determinations can be further increased by studying the elevation angle measurements for specific propagation paths to artificially-induced backscatter from the EISCAT Troms $\varnothing$ (Rietveld et al., 1993) and SPEAR (Wright et al., 2000; Robinson et al., 2006) ionospheric heating facilities.

There is an additional complication when mapping scattering locations that needs to be considered. Due to the cone angle effect (e.g. Milan et al., 1997; André et al., 1998) the azimuth angle that the propagation path of a beam makes with the radar boresite varies with the elevation angle. This is dealt with in the present SuperDARN software by determining an effective elevation angle for the virtual propagation path at each range based on the measured range and the model virtual height for that range. The direction of the beam at each range is then adjusted based on this effective elevation angle. For our new empirical virtual height model, where we use the pseudo virtual height in the $1 \frac{1}{2}$-hop F-region propagation region, the effective elevation angle of the pesudo virtual path will be different to the effective elevation angle of the true $1 \frac{1}{2}$-hop virtual path. Hence the azimuth angle at far ranges will be in error if this issue is not taken into account. Correcting for this factor will probably require more sophisticated changes to the present mapping software.

Finally, to increase our confidence in the reliability of the model it is important to test it with an independent data set, i.e. using data from another SuperDARN radar not used in the determination of the model. In addition, definite knowledge of the locations of irregularities on the expected propagation path will increase the reliability of any such test. The companion paper (Yeoman et al., 2008) studies data from the two SuperDARN radars that form the CUTLASS pair, from times when ionospheric irregularities have been artificially induced by an ionospheric heating facility. Both the EISCAT Troms $\varnothing$ (Rietveld et al., 1993) and SPEAR (Wright et al., 2000; Robinson et al., 2006) ionospheric heating facilities are located within the fields of view of both the CUTLASS Finland and CUTLASS Iceland East SuperDARN radars, and this study includes data from heating campaigns throughout the lifetime of SuperDARN.

\section{Summary}

We have developed a new empirical virtual height model for SuperDARN HF radar backscatter by studying elevation angle data from 5 years of backscatter from the Saskatoon SuperDARN radar. Our new virtual height model reduces the systematic uncertainties in the mapping of the locations of scattering regions, especially at far ranges (typically by $\sim 200 \mathrm{~km}$ ).

Acknowledgements. The authors would like to thank M. Freeman for helpful discussions.

Editor in Chief W. Kofman thanks A. V. Koustov and J. M. Ruohoniemi for their help in evaluating this paper.

\section{References}

André, D., Sofko, G. J., Baker, K., and MacDougall, J.: SuperDARN interferometry: Meteor echoes and electron densities from groundscatter, J. Geophys. Res., 103, 7003-7015, 1998. 
André, R., Hanuise, C., Villain, J.-P., and Cerisier, J.-C.: HF radars: Multifrequency study of refraction effects and localization of scattering, Radio Sci., 32, 153-168, 1997.

Baker, K. B., Greenwald, R. A., Walker, A. D. M., Bythrow, P. F., Zanetti, L. J., Potemra, T. A., Hardy, D. A., Rich, F. J., and Rino, C. L.: A case study of plasma processes in the dayside cleft, J. Geophys. Res., 91, 3130-3144, 1986.

Baker, K. B., Dudeney, J. R., Greenwald, R. A., Pinnock, M., Newell, P. T., Rodger, A. S., Mattin, N., and Meng, C.-I.: HF radar signatures of the cusp and low-latitude boundary layer, J. Geophys. Res., 100, 7671-7695, 1995.

Bates, H. F. and Albee, P. R.: Aspect sensitivity of $F$ layer HF backscatter echoes, J. Geophys. Res., 75, 165-170, 1970.

Breit, G. and Tuve, M. A.: A test of the existence of the conducting layer, Phys. Rev., 28, 554-575, 1926.

Chisham, G., Pinnock, M., and Rodger, A. S.: Poleward-moving HF radar flow bursts in the cusp: Transient changes in flow speed or direction?, Geophys. Res. Lett., 27, 919-922, 2000.

Chisham, G., Freeman, M. P., Sotirelis, T., Greenwald, R. A., Lester, M., and Villain, J.-P.: A statistical comparison of SuperDARN spectral width boundaries and DMSP particle precipitation boundaries in the morning sector ionosphere, Ann. Geophys., 23, 733-743, 2005,

http://www.ann-geophys.net/23/733/2005/.

Chisham, G., Lester, M., Milan, S. E., Freeman, M. P., Bristow, W. A., Grocott, A., McWilliams, K. A., Ruohoniemi, J. M., Yeoman, T. K., Dyson, P. L., Greenwald, R. A., Kikuchi, T., Pinnock, M., Rash, J. P. S., Sato, N., Sofko, G. J., Villain, J.-P., and Walker, A. D. M.: A decade of the Super Dual Auroral Radar Network (SuperDARN): Scientific achievements, new techniques and future directions, Surv. Geophys., 28, 33-109, 2007.

Greenwald, R. A., Baker, K. B., Dudeney, J. R., Pinnock, M., Jones, T. B., Thomas, E. C., Villain, J.-P., Cerisier, J.-C., Senior, C., Hanuise, C., Hunsucker, R. D., Sofko, G., Koehler, J., Nielsen, E., Pellinen, R., Walker, A. D. M., Sato, N., and Yamagishi, H.: DARN/SuperDARN: A global view of the dynamics of highlatitude convection, Space Sci. Rev., 71, 761-796, 1995.

Hanuise, C., Villain, J.-P., Gresillon, D., Cabrit, B., Greenwald, R. A., and Baker, K. B.: Interpretation of HF radar ionospheric Doppler spectra by collective wave scattering theory, Ann. Geophys., 11, 29-39, 1993, http://www.ann-geophys.net/11/29/1993/.

Huang, C.-S., André, D., and Sofko, G. J.: High-latitude ionospheric perturbations and gravity waves 1 . Observational results, J. Geophys. Res., 103, 2131-2141, 1998a.

Huang, C.-S., André, D., and Sofko, G. J.: High-latitude ionospheric perturbations and gravity waves 2 . Numerical simulations, J. Geophys. Res., 103, 2143-2153, 1998b.

Huang, C.-S., Murr, D., Sofko, G. J., Hughes, W. J., and Moretto, T.: Ionospheric convection response to changes of interplanetary magnetic field $B_{z}$ component during strong $B_{y}$ component, J. Geophys. Res., 105, 5231-5243, 2000.

Hubert, B., Milan, S. E., Grocott, A., Cowley, S. W. H., and Grard, J.-C.: Dayside and nightside reconnection rates inferred from IMAGE-FUV and Super Dual Auroral Network data, J. Geophys. Res., 111, A03217, doi:10.1029/2005JA011140, 2006.
Jones, R. M. and Stephenson, J. J.: A versatile three-dimensional ray tracing program for radio waves in the ionosphere, Rep. 7576, Office of Telecommun., US Dep. of Commerce, Boulder, Colorado, 1975.

Milan, S. E., Jones, T. B., Robinson, T. R., Thomas, E. C., and Yeoman, T. K.: Interferometric evidence for the observation of ground backscatter originating behind the CUTLASS coherent HF radars, Ann. Geophys., 15, 29-39, 1997, http://www.ann-geophys.net/15/29/1997/.

Rietveld, M. T., Kohl, H., Kopka, H., and Stubbe, P.: Introduction to ionospheric heating at Troms $\varnothing$, I, Experimental overview, J. Atmos. Terr. Phys., 55, 577-599, 1993.

Robinson, T. R., Yeoman, T. K., Dhillon, R. S., Lester, M., Thomas, E. C., Thornhill, J. D., Wright, D. M., van Eyken, A. P., and McCrea, I. W.: First observations of SPEAR-induced artificial backscatter from CUTLASS and the EISCAT Svalbard radars, Ann. Geophys., 24, 291-309, 2006, http://www.ann-geophys.net/24/291/2006/.

Ruohoniemi, J. M., Greenwald, R. A., Baker, K. B., Villain, J.-P., and McCready, M. A.: Drift motions of small-scale irregularities in the high-latitude $F$ region: An experimental comparison with plasma drift motions, J. Geophys. Res., 92, 4553-4564, 1987.

Senior, A., Borisov, N. D., Kosch, M. J., Yeoman, T. K., Honary, F., and Rietveld, M. T.: Multi-frequency HF radar measurements of artificial F-region field-aligned irregularities, Ann. Geophys., 22, 3503-3511, 2004,

http://www.ann-geophys.net/22/3503/2004/.

Vickrey, J. F., Rino, C. L., and Potemra, T. A.: Chatanika/Triad observations of unstable ionization enhancements in the auroral $F$-region, Geophys. Res. Lett., 7, 789-792, 1980.

Villain, J.-P., Greenwald, R. A., and Vickrey, J. F.: HF ray tracing at high latitudes using measured meridional electron density distributions, Radio Sci., 19, 359-374, 1984.

Villain, J.-P., Hanuise, C., and Caudal, G.: A SAFARI-EISCAT comparison between the velocity of $\mathrm{F}$ region small-scale irregularities and the ion drift, J. Geophys. Res., 90, 8433-8443, 1985.

Weaver, P. F.: Backscatter echoes from field-aligned irregularities in the $F$ region, J. Geophys. Res., 70, 5425-5432, 1965.

Woodfield, E. E., Davies, J. A., Lester, M., Yeoman, T. K., Eglitis, P., and Lockwood, M.: Nightside studies of coherent HF radar spectral width behaviour, Ann. Geophys., 20, 1399-1413, 2002, http://www.ann-geophys.net/20/1399/2002/.

Wright, D. M., Davies, J. A., Robinson, T. R., Chapman, P. J., Yeoman, T. K., Thomas, E. C., Lester, M., Cowley, S. W. H., Stocker, A. J., Horne, R. B., and Honary, F.: Space Plasma Exploration by Active Radar (SPEAR): an overview of a future radar facility, Ann. Geophys., 18, 1248-1255, 2000, http://www.ann-geophys.net/18/1248/2000/.

Yeoman, T. K., Wright, D. M., Stocker, A. J., and Jones, T. B.: An evaluation of range accuracy in the Super Dual Auroral Radar Network over-the-horizon HF radar systems, Radio Sci., 36, 801-813, 2001.

Yeoman, T. K., Chisham, G., Baddeley, L. J., Dhillon,, R. S., Karhunen, T. J. T., Robinson, T. R., Senior, A., and Wright, D. M.: Mapping ionospheric backscatter measured by the SuperDARN HF radars - Part 2: Assessing SuperDARN virtual height models, Ann. Geophys., 26, 843-852, 2008, http://www.ann-geophys.net/26/843/2008/. 